\title{
Calculation of Stress Intensity Factors for Interfacial Cracks in Fiber Metal Laminates
}

\author{
John T. Wang* \\ NASA Langley Research Center, Hampton, VA 23681
}

\begin{abstract}
Stress intensity factors for interfacial cracks in Fiber Metal Laminates (FML) are computed by using the displacement ratio method recently developed by Sun and Qian (1997, Int. J. Solids. Struct. 34, 2595-2609). Various FML configurations with single and multiple delaminations subjected to different loading conditions are investigated. The displacement ratio method requires the total energy release rate, bimaterial parameters, and relative crack surface displacements as input. Details of generating the energy release rates, defining bimaterial parameters with anisotropic elasticity, and selecting proper crack surface locations for obtaining relative crack surface displacements are discussed in the paper. Even though the individual energy release rates are nonconvergent, mesh-sizeindependent stress intensity factors can be obtained. This study also finds that the selection of reference length can affect the magnitudes and the mode mixity angles of the stress intensity factors; thus, it is important to report the reference length used with the calculated stress intensity factors.
\end{abstract}

\section{Introduction}

$\mathrm{T}$ HE NASA Aircraft Aging and Durability Program is currently supporting research efforts in the fracture and fatigue of Fiber Metal Laminates (FMLs). FMLs, such as Glare which contains glass fiber layers (about 0.005 in/layer) and thin aluminum layers (about 0.016 in/layer), and CentrAL which contains Glare sublaminates in the middle and thicker outer aluminum layers ${ }^{1}(0.32$ in. or 0.16 in.), are being investigated. Schematics of the Glare and the CentrAL material systems are shown in Fig. 1. Note that GLARE ${ }^{\circledR}$ is a registered trademark of Structural Laminates Company, a joint venture of Aluminum Corporation of America (ALCOA) and Akzo Nobel to produce Glare; and CentrAL is a patent held by Alcoa, GTM Advanced Structures, and Delft University of Technology. Glare has been found to possess excellent fatigue and damage tolerance properties. Due to the presence of the fiber layers, the fatigue crack growth rate for Glare is much less than that for monolithic aluminum. Unfortunately, the manufacturing cost, related to milling, pretreatment, and storage of thin aluminum sheets and the labor intensive layup process, limits the configurations of Glare to approximately 6/5 lay-ups (6 metal sheets and 5 fiber prepreg layers in between). ${ }^{1}$ Consequently, Glare is mainly used for fuselage panel applications, and cannot be used for wing box skins which require large material thickness. New CentrAL hybrid concepts ${ }^{1}$, which bond thicker outer aluminum layers to Glare, are being developed by Alcoa, Inc. and its partners to reduce manufacturing cost and satisfy fatigue and damage tolerance requirements of the thick lower wing skin panel applications. Research in fracture and fatigue of CentrAL FMLs is being conducted at NASA Langley Research Center under a Space Act Agreement between NASA and ALCOA.

Delamination failure can significantly affect the fatigue and damage tolerance behaviors of FMLs. Delaminations often occur at the interfaces between metal layers and fiber layers. The crack growth behavior in an aluminum layer greatly depends on the delamination shape around (surrounding) the crack. ${ }^{1-3}$ To accurately predict the interfacial delamination growth in FMLs, an appropriate failure criterion needs to be established. ${ }^{3}$ Due to the oscillatory characteristic of stress and displacement fields near the crack tip, the energy release rates of individual modes are not convergent with mesh refinement ${ }^{4,5}$ thus, establishing the delamination failure criterion for FML interfacial cracks based on the individual mode of energy release rates may be problematic. On the other hand, the total energy release rate $G_{T}$ and the stress intensity factors of individual modes are well defined and are expected to be independent of mesh size. ${ }^{4,6-8}$ Thus, the ratios between the stress intensity factors, $K_{I I} / K_{I}$ and $K_{I I I} / K_{I}$, and the

\footnotetext{
* Aerospace Engineer, DDTRB/RTD, NASA Langley Research Center, MS188E, Hampton, VA 23681, AIAA Associate Fellow
} 
mode mixity angles, $\psi_{12}=\tan ^{-1}\left(K_{I I} / K_{I}\right)$ and $\psi_{13}=\tan ^{-1}\left(K_{I I I} / K_{I}\right)$, are also expected to be independent of mesh size. Once the mode mixity angles are determined, the total energy release rate can be used to define the mixed mode failure criterion. ${ }^{3,7,8}$

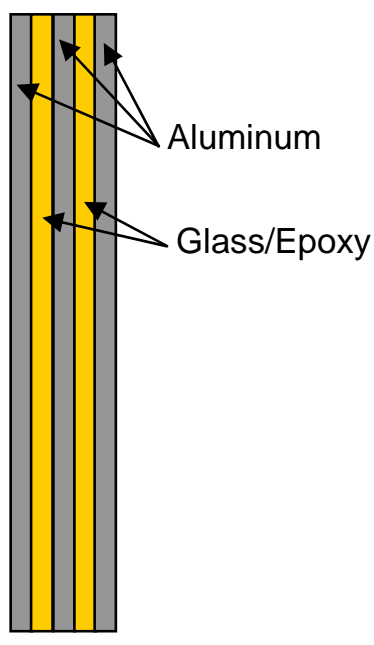

(a) Glare

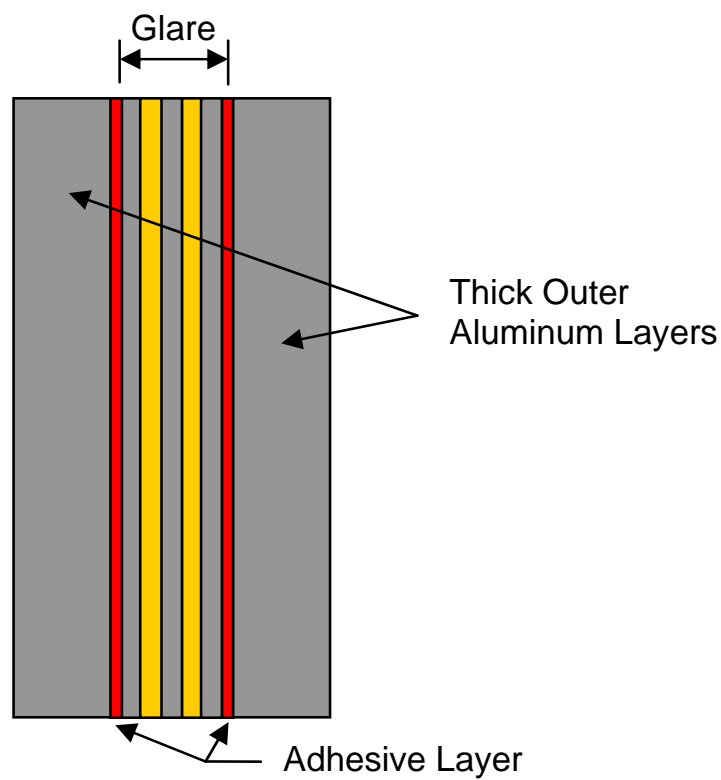

(b) CentrAL

Fig. 1 Schematics of Glare and CentrAL cross-sections.

The objective of this paper is to demonstrate that mesh-size-independent stress intensity factors are obtainable and can be efficiently computed for delamination cracks in FMLs. The stress intensity factors are computed with the displacement ratio method developed by Sun and Qian. ${ }^{7,8}$ The inputs for the displacement ratio method are the total strain energy release rate, material parameters from anisotropic elasticity, and the near-tip relative crack surface displacements.

This paper consists of four major sections. Section II describes background information related to the definition of the stress intensity factors, the explicit relationships between the near-tip stress fields and the stress intensity factors, and the explicit relationships between the displacement fields and the stress intensity factors. The Stroh formalism $^{9}$ of anisotropic elasticity used in establishing these relationships is given in the Appendix. The displacement ratio method for obtaining individual modes of stress intensity factors ${ }^{7,8}$ is introduced next in this section. At the end of this section, a mixed mode fracture criterion is given to set the critical condition for an interfacial crack to propagate. In Section III, the stress intensity factors for delaminated FMLs subjected to the opening mode loading and the in-plane tensile loading are computed. Finite element models of FMLs with interfacial cracks are created and analyzed to provide the nodal forces and displacements needed for the Virtual Crack Closure Technique (VCCT) ${ }^{10}$ to obtain the energy release rates. The relative crack surface displacements and the total energy release rates are then used for the displacement ratio method to compute the stress intensity factors. Lessons learned such as how to avoid a potential pitfall in using the displacement ratio method and how to properly present the stress intensity factors are also given. Finally, Section IV gives concluding remarks and summarizes the findings of this study.

\section{Background Information}

In this section, relevant equations for computing the stress intensity factors for interfacial cracks in composite materials presented in Qian and Sun's paper ${ }^{8}$ are summarized. These equations are used for defining stress intensity factors, characterizing the near-tip stress and displacement fields, and presenting the displacement ratio method. All materials presented in this section can be applied for investigating the interfacial cracks in FMLs. 


\section{A. Stress Intensity Factors Definition}

The stress intensity factors definition for interfacial crack between two dissimilar anisotropic materials proposed by $\mathrm{Hwu}^{11}$ and $\mathrm{Wu}^{12}$ is used in this study. For generalized plane strain problems, such as the representative FML under an opening mode loading shown in Fig. 2, in which the strains are independent of the coordinate $x_{3}$, the stress intensity factors can be expressed as

$$
\left\{\begin{array}{c}
K_{I I} \\
K_{I} \\
K_{I I I}
\end{array}\right\}=\lim _{r \rightarrow 0} \sqrt{2 \pi r} \boldsymbol{\Lambda}\left\langle\left\langle(r / \hat{r})^{-i \varepsilon_{\alpha}}\right\rangle\right\rangle \boldsymbol{\Lambda}^{-1}\left\{\begin{array}{c}
\sigma_{12} \\
\sigma_{22} \\
\sigma_{23}
\end{array}\right\}
$$

where the angular bracket $\langle\langle\rangle$.$\rangle represents a 3x3 diagonal matrix. \varepsilon_{\alpha}(\alpha=1,2,3)$ are constants related to the oscillation index $\varepsilon$ (see Appendix), and $\Lambda$ is the eigenvector matrix associated with an eigenequation, Eq. (A18), established by using the Stroh formalism ${ }^{9}$ as discussed in the Appendix. $\hat{r}$ is an arbitrary chosen reference length parameter. ${ }^{11-13}$ Stress intensity factors obtained for one reference length parameter can be easily converted to another reference length parameter ${ }^{8}$. For example, if the reference length parameter $\hat{r}$ is chosen to be the crack length $2 a$, stress intensity factors for another $\hat{r}$ can be obtained by

$$
\mathbf{K}(\hat{r})=\Lambda\left\langle\left\langle(2 a / \hat{r})^{-i \varepsilon_{\alpha}}\right\rangle\right\rangle \boldsymbol{\Lambda}^{-1} \mathbf{K}(2 a)
$$

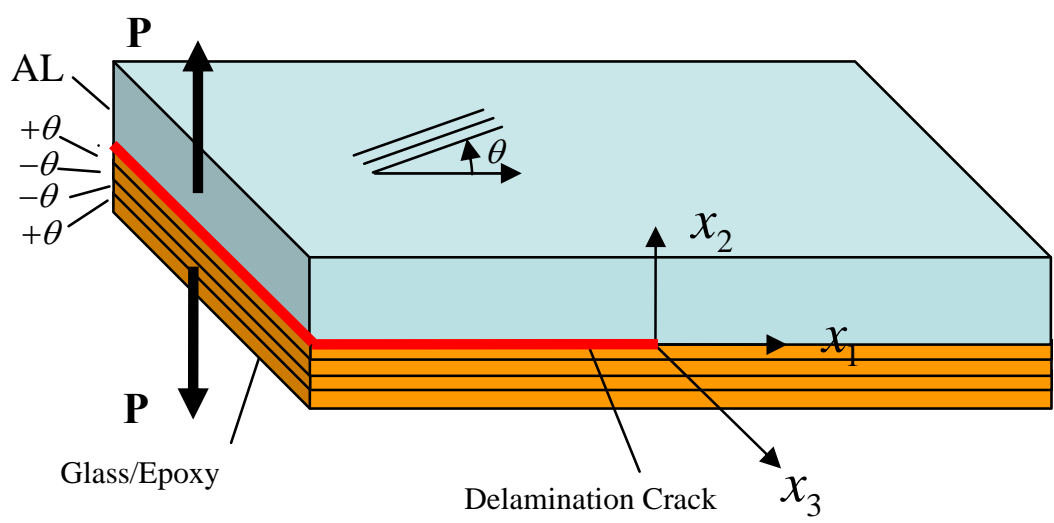

Fig. 2 A representative fiber metal laminate.

The stress intensity factors defined in Eq. (1) have the following properties:

1. The units of the stress intensity factors, $(p s i \times \sqrt{i n})$, are consistent with the conventional stress intensity factors.

2. The stress intensity factors defined can be reduced to the classical stress intensity factors for a crack tip in homogeneous material.

3. When the applied load is scaled by a factor, all intensity factors increase by the same factor.

4. $K_{I I}, K_{I}$, and $K_{I I I}$ represent the intensity of singularity in the directions of $x_{1}, x_{2}$, and $x_{3}$, respectively. ${ }^{11}$

5. Unlike the energy release rates computed by the VCCT, the stress intensity factors for interfacial cracks are convergent and not dependent on the virtual crack extension length (mesh size) $\Delta a$. Due to the multiplication of $\left\langle\left\langle(r / \hat{r})^{-i \varepsilon_{\alpha}}\right\rangle\right\rangle$ in Eq. (1), the individual stress intensity factors are well defined and non-oscillatory, as evidenced by the explicit forms of the individual mode of stress intensity factors, which do not contain oscillatory terms similar to $(\Delta a)^{i \varepsilon}$ (Refs. 4 and 8). 


\section{B. Characterizing Near Tip Fields}

Based on the stress intensity factors defined in Eq. (1), $\mathrm{Hwu}^{11}$ found that the near-tip stresses and the near-tip relative crack surface displacements for an interfacial delamination crack can be expressed as

$$
\left\{\begin{array}{l}
\sigma_{12} \\
\sigma_{22} \\
\sigma_{23}
\end{array}\right\}=\frac{1}{\sqrt{2 \pi r}} \boldsymbol{\Lambda}\left\langle\left\langle(r / \hat{r})^{i \varepsilon_{\alpha}}\right\rangle\right\rangle \boldsymbol{\Lambda}^{-1}\left\{\begin{array}{c}
K_{I I} \\
K_{I} \\
K_{I I I}
\end{array}\right\}
$$

and

$$
\left\{\begin{array}{l}
\Delta u_{1} \\
\Delta u_{2} \\
\Delta u_{3}
\end{array}\right\}=\sqrt{\frac{2 r}{\pi}}\left(\bar{\Lambda}^{T}\right)^{-1} \times\left\langle\left\langle\frac{(r / \hat{r})^{i \varepsilon_{\alpha}}}{\left(1+2 i \varepsilon_{\alpha}\right) \cosh \left(\pi \varepsilon_{\alpha}\right)}\right\rangle\right\rangle \boldsymbol{\Lambda}^{-1}\left\{\begin{array}{c}
K_{I I} \\
K_{I} \\
K_{I I I}
\end{array}\right\}
$$

Based on the material parameters presented in the Appendix, the near tip stresses in Eq. (3) for interfacial cracks in laminated composites can be explicitly written as

$$
\begin{aligned}
& \sigma_{12}=\frac{1}{\sqrt{2 \pi r}}\left[\frac{a_{f}}{b_{f}} \operatorname{Im}\left(\xi_{1}\right) K_{I}+\left(\frac{a_{f}}{g_{f}} W_{21} \operatorname{Re}\left(\xi_{1}\right)+\frac{d_{f}}{g_{f}} W_{32}\right) K_{I I}-\frac{a_{f}}{g_{f}} W_{32}\left(\operatorname{Re}\left(\xi_{1}\right)-1\right) K_{I I I}\right] \\
& \sigma_{22}=\frac{1}{\sqrt{2 \pi r}}\left[\operatorname{Re}\left(\xi_{1}\right) K_{I}-\frac{b_{f}}{g_{f}} W_{21} \operatorname{Im}\left(\xi_{1}\right) K_{I I}+\frac{b_{f}}{g_{f}} W_{32} \operatorname{Im}\left(\xi_{1}\right) K_{I I I}\right] \\
& \sigma_{23}=\frac{1}{\sqrt{2 \pi r}}\left[-\frac{d_{f}}{b_{f}} \operatorname{Im}\left(\xi_{1}\right) K_{I}-\frac{d_{f}}{g_{f}} W_{21}\left(\operatorname{Re}\left(\xi_{1}\right)-1\right) K_{I I}+\left(\frac{d_{f}}{g_{f}} W_{32} \operatorname{Re}\left(\xi_{1}\right)+\frac{a_{f}}{g_{f}} W_{21}\right) K_{I I I}\right],
\end{aligned}
$$

and the relative crack surface displacements in Eq. (4) can be explicitly written as

$$
\begin{aligned}
& \Delta u_{1}=\sqrt{\frac{2 r}{\pi}}\left[\frac{b_{f}^{2} c_{f}^{2}}{2 g_{f}^{2}} W_{21} \operatorname{Re}\left(\xi_{2}\right) H_{1}+\frac{d_{f} b_{f}}{g_{f}^{2}} \beta D_{22} H_{2}+\frac{b_{f} c_{f}^{2}}{2 g_{f}} W_{21} \operatorname{Im}\left(\xi_{2}\right) K_{I}\right] \\
& \Delta u_{2}=\sqrt{\frac{2 r}{\pi}}\left[\frac{c_{f}^{2}}{2} \operatorname{Re}\left(\xi_{2}\right) K_{I}-\frac{b_{f} c_{f}^{2}}{2 g_{f}} \operatorname{Im}\left(\xi_{2}\right) H_{1}\right] \\
& \Delta u_{3}=\sqrt{\frac{2 r}{\pi}}\left[\frac{b_{f}^{2} c_{f}^{2}}{2 g_{f}^{2}} W_{32} \operatorname{Re}\left(\xi_{2}\right) H_{1}+\frac{a_{f} b_{f}}{g_{f}^{2}} \beta D_{22} H_{2}-\frac{b_{f} c_{f}^{2}}{2 g_{f}} W_{32} \operatorname{Im}\left(\xi_{2}\right) K_{I}\right]
\end{aligned}
$$

where $W_{21}$ and $W_{32}$ are elements of the $\mathbf{W}$ defined in Eq. (A17) in the Appendix. $a_{f}, b_{f}, c_{f}$, and $d_{f}$ are also defined in the Appendix. $g_{f}, H_{1}, H_{2}$, and complex constants $\xi_{1}, \xi_{2}$ are defined as

$g_{f}=a_{f} W_{21}+d_{f} W_{32}, H_{1}=W_{21} K_{I I}-W_{32} K_{I I I}, H_{2}=d_{f} K_{I I}+a_{f} K_{I I I}$,

$\xi_{1}=(r / \hat{r})^{i \varepsilon}, \xi_{2}=\frac{\xi_{1}}{(1+2 i \varepsilon) \cosh (\pi \varepsilon)}$

where $\varepsilon$ is the oscillation index defined in the Appendix.

\section{Displacement Ratio Method for Mode Separation}

The displacement ratio method ${ }^{7,8}$ is based on the total energy release rate and the ratios of the near-tip crack surface displacements, obtained from finite element analysis results, to compute the stress intensity factors. The total 
energy release rate can be computed by the VCCT. The following equation shows that $G_{T}$ can be expressed in terms of the stress intensity factors. ${ }^{8}$

$$
\begin{aligned}
G_{T} & =\lim _{\Delta a \rightarrow 0}\left(G_{I}+G_{I I}+G_{I I I}\right) \\
& =\frac{1}{4}\left[\frac{D_{22}}{\cosh ^{2}(\pi \varepsilon)} K_{I}^{2}+\left(D_{11}-\frac{W_{21}^{2}}{D_{22}}\right) K_{I I}^{2}+\left(D_{33}-\frac{W_{32}^{2}}{D_{22}}\right) K_{I I I}^{2}+2\left(\frac{W_{21} W_{32}}{D_{22}}+D_{13}\right) K_{I I} K_{I I I}\right]
\end{aligned}
$$

The total energy release rate equation is established by using the tip stresses and displacements in Eqs. (5) and (6) in Irwin's crack closure integrals. ${ }^{14}$

The near tip relative crack surface displacement can be obtained from the same finite element analysis used for the VCCT. Based on Eq. (6), these crack surface displacements can be related to the stress intensity factors as

$$
\begin{aligned}
& \Delta u_{1}=c_{1} K_{I}+c_{2} K_{I I}+c_{3} K_{I I I} \\
& \Delta u_{2}=d_{1} K_{I}+d_{2} K_{I I}+d_{3} K_{I I I} \\
& \Delta u_{3}=e_{1} K_{I}+e_{2} K_{I I}+e_{3} K_{I I I}
\end{aligned}
$$

For brevity, the explicit expressions of coefficients $c_{i}, d_{i}$, and $e_{i}(i=1,2,3)$ are not presented here. From Eq. 8, the ratios $K_{I I} / K_{I}$ and $K_{I I I} / K_{I}$ can be determined by the displacement ratios $\Delta u_{2} / \Delta u_{1}$ and $\Delta u_{3} / \Delta u_{1}$. Finally, these stress intensity factor ratios are used in Eq. 7 to obtain the $K_{I}, K_{I I}$, and $K_{I I I}$. The stress intensity factors can be obtained very efficiently by the displacement ratio method because the inputs for the displacement ratio method, the total energy release rate and the relative crack surface displacements, can be provided by a single finite element analysis.

\section{Failure Criterion}

The ratios of individual stress intensity factors, obtained by the displacement ratio method, are used to define the mode mixity angles $\psi_{12}$ and $\psi_{13}$. Here, $\tan \psi_{12}=K_{I I} / K_{I}$ and $\tan \psi_{13}=K_{I I I} / K_{I}$. The critical condition for an interfacial crack to propagate can be given by a mixed mode fracture criterion

$$
G_{T}\left(\psi_{12}, \psi_{13}\right)=G_{C}\left(\psi_{12}, \psi_{13}\right)
$$

where $G_{C}$ is the critical energy release rate (fracture toughness) that needs to be determined experimentally. Several specimens are available for this purpose such as the tensile sandwich test specimens ${ }^{15,16}$ and the four point bend specimens. ${ }^{17} \psi_{12}$ and $\psi_{13}$ are the model mixity angles, defined using an arbitrary reference parameter $\hat{r}$. Examples of $\psi_{12}$ and $\psi_{13}$ as a function of $\hat{r}$ are given in Section III.B.

According to Ref. 18, the following steps may be taken to apply the mixed mode fracture criterion for assessing the interfacial crack failure in FMLs: (i) measure the fracture toughness $G_{C}$ of the interface crack as a function of mode mixity angle; (ii) calculate energy release rate $G_{T}$ and mode mixity for the interface crack in the FML component, and (iii) apply the fracture criterion to assess the failure load of the FML component.

\section{Stress Intensity Factors for Interfacial Cracks in FMLs}

FMLs with delamination cracks were analyzed to demonstrate that mesh-size-independent stress intensity factors can be easily and efficiently obtained by the displacement ratio method. Finite element analyses were performed to obtain the relative crack surface displacements and the nodal forces needed for using VCCT to compute the total energy release rate. These relative crack surface displacements and the total energy release rates are then used in the displacement ratio method to compute the stress intensity factors. Two FML configurations were used in this study. The first one, studied in Section III.A, is a representative fiber metal laminate with an interfacial delamination 
between metal and fiber layers, and the second one, studied in Section III.B, is a CentrAL-like FML with multiple interfacial delaminations.

The first FML configuration subjected to an opening mode loading was designed to test whether mesh size independent stress intensity factors can always be obtained for interfacial cracks in FMLs. Plane strain analyses using ABAQUS ${ }^{\odot}$ 2D elements were performed for models with principal material directions aligned with the specimen's $x_{1}$ and $x_{3}$ axes (see Fig. 3) and generalized plane strain analyses using ABAQUS ${ }^{\odot}$ 3D elements were performed for models with principal material directions not aligned with the axes.

The second FML configuration subjected to an in-plane tensile loading was designed to investigate the variations of stress intensity factors with delamination locations in a CentrAL-like FML with multiple interfacial delaminations under an in-plane tensile loading. The effects of the reference length parameter in stress intensity factors and mode mixity angles were also investigated. These computed stress intensity factors and mode mixity angles could be used in the interfacial failure criterion in Eq. (9) to determine the interfacial crack propagations.

Material properties for the fiber and the aluminum layers of the FMLs are listed in Table 1. These properties are obtained from Ref. 19.

Table 1 Material Properties of Unidirectional Glass/Epoxy and Aluminum Layers.

\begin{tabular}{|l|l|l|l|}
\hline \multicolumn{2}{|l|}{ Glass/Epoxy } & Aluminum \\
\hline$E_{1}(\mathrm{msi})$ & 7.977 & $E$ (msi) & 10.5 \\
\hline$E_{2}(\mathrm{msi})$ & 1.378 & $v$ & 0.33 \\
\hline$E_{3}(\mathrm{msi})$ & 1.378 & & \\
\hline$v_{12}$ & 0.33 & & \\
\hline$v_{13}$ & 0.33 & & \\
\hline$v_{23}$ & 0.45 & & \\
\hline$G_{12}(\mathrm{msi})$ & 0.8 & & \\
\hline$G_{13}(\mathrm{msi})$ & 0.8 & & \\
\hline$G_{23}(\mathrm{msi})$ & 0.435 & & \\
\hline
\end{tabular}

\section{A. Representative Fiber Metal Laminate under Opening Mode Loading}

A representative fiber metal laminate with a delamination under an opening mode loading shown in Fig. 2 was analyzed to demonstrate that mesh-size-independent stress intensity factors can be computed using the displacement ratio method. The delamination is located at the interface between the upper aluminum part and the lower four-layer Glass/Epoxy part. The lay-ups of the Glass/Epoxy part include $\left[0_{4}\right],[+15 /-15]_{\mathrm{S}},[+30 /-30]_{\mathrm{S}},[+45 /-45]_{\mathrm{S}},[+60 /-60]_{\mathrm{S}}$, $[+75 /-75]_{S}$, and $\left[90_{4}\right]$. Only linear analyses were performed in this study. The thickness of the aluminum is 2.0 in. and the thickness of each fiber layer is 0.5 in. to assure high bending stiffnesses for both crack flanks to avoid geometrically nonlinear deformations. The delamination crack length is $10 \mathrm{in}$. and an equal and opposite vertical load $\mathrm{P}$ of $100 \mathrm{lb} / \mathrm{in}$ (distributed load in $x_{3}$ direction) is applied at the left end of each crack flank. The right end of the model is fixed. Plane strain and generalized plane strain finite element models, using ABAQUS ${ }^{\odot}$ CPE8 and C3D8I elements, respectively, were created and analyzed with ABAQUS ${ }^{\odot} /$ Standard. $^{20}$ Plane strain models using 2D CPE8 elements were created for FMLs with $\left[0_{4}\right]$ and $\left[90_{4}\right]$ Glass/Epoxy lay-ups, and generalized plane strain models using 3D C3D8I elements were created for all seven types of Glass/Epoxy layups to properly input the off-axis Glass/Epoxy material properties. The VCCT is used to obtain the energy release rates. The total energy release rate, obtained by summing the individual mode of energy release rates, is then used in the displacement ratio method for calculating the stress intensity factors. 


\section{Plane Strain Finite Element Analyses}

Four plane strain finite element models, representing three different crack tip mesh sizes, $\Delta a=0.0125,0.025$, 0.05 , and 0.1 in., were created (see the inset in Fig. 3). Two ABAQUS ${ }^{\odot}$ finite element analyses were performed. The first analysis assumed the glass fibers were laid up in a $\left[\mathrm{O}_{4}\right]$ stack, and the second analysis assumed the glass fibers were laid up in a $\left[90_{4}\right]$ stack.

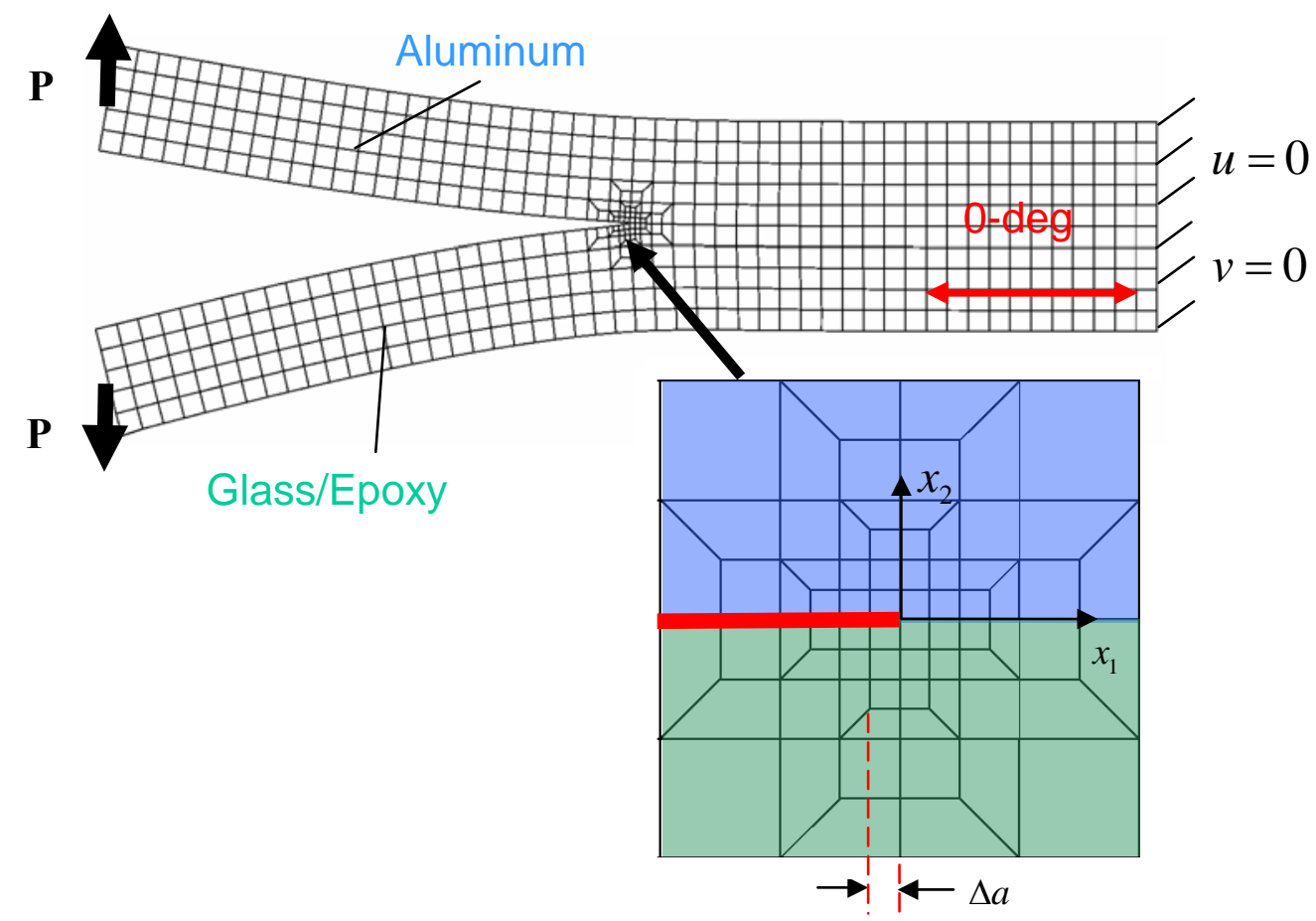

Fig. 3 Typical plane strain finite element model.

The finite element model with fiber in the 0-deg direction is shown in Fig. 3. The energy release rates, obtained by the VCCT, for the models with glass fibers in the 0-deg direction are plotted in Fig. 4. The opening mode energy release rate $G_{I}$ and the sliding mode energy release rate $G_{I I}$ are nonconvergent with increased mesh refinement, but the total energy release rate $G_{T}$ is well defined and independent of the crack tip mesh size (i.e. decreasing $\Delta a$ ).

The individual stress intensity factors for the models with various $\Delta a$ were computed with the displacement ratio method, using two different reference length parameters ( $\hat{r}=2$ and 20 in). Note that the relative crack surface displacements for the displacement ratio method are taken at one element away from the crack tip. Figure 5 shows that the individual stress intensity factors computed are nearly constant for crack tip mesh sizes $\Delta a \geq 0.025$ in. The stress intensity factor $K_{I I}$ at $\Delta a=0.0125$ in. is less than those of larger mesh sizes. This may be due to the fact that the oscillatory displacement fields near the crack tip cannot be accurately modeled with the quadratic ABAQUS ${ }^{\odot}$ CPE8 elements. It is reasonable to hypothesize that the relative crack surface displacements at a location further away from the crack tip may be better predicted by ABAQUS ${ }^{\odot}$. To test this hypothesis, the relative crack opening displacements were taken at the location 0.025 in. (two elements) away from the crack tip for computing the stress intensity factors for the model of $\Delta a=0.0125 \mathrm{in}$. The stress intensity values of the smallest mesh size model are increased to be in line with those of the larger mesh size models as shown in Fig. 6. This indicates that mesh-sizeindependent stress intensity factors can be obtained if the relative crack opening displacements are not taken very near the crack tip.

Furthermore, Figures 5 and 6 show that changing the reference length from $\hat{r}=2$ to $\hat{r}=20$ in. can result in changes of both $K_{I}$ and $K_{I I}$ values. For this case, the changes are non-proportional, a larger reduction occurs in the $K_{I I}$ values than in the $K_{I}$ values. The non-proportional changes indicate that changing the reference length not only changes the magnitudes of $K_{I}$ and $K_{I I}$ but also their mode mixity angle, $\psi_{12}=\tan ^{-1}\left(K_{I I} / K_{I}\right)$. Fortunately, the 


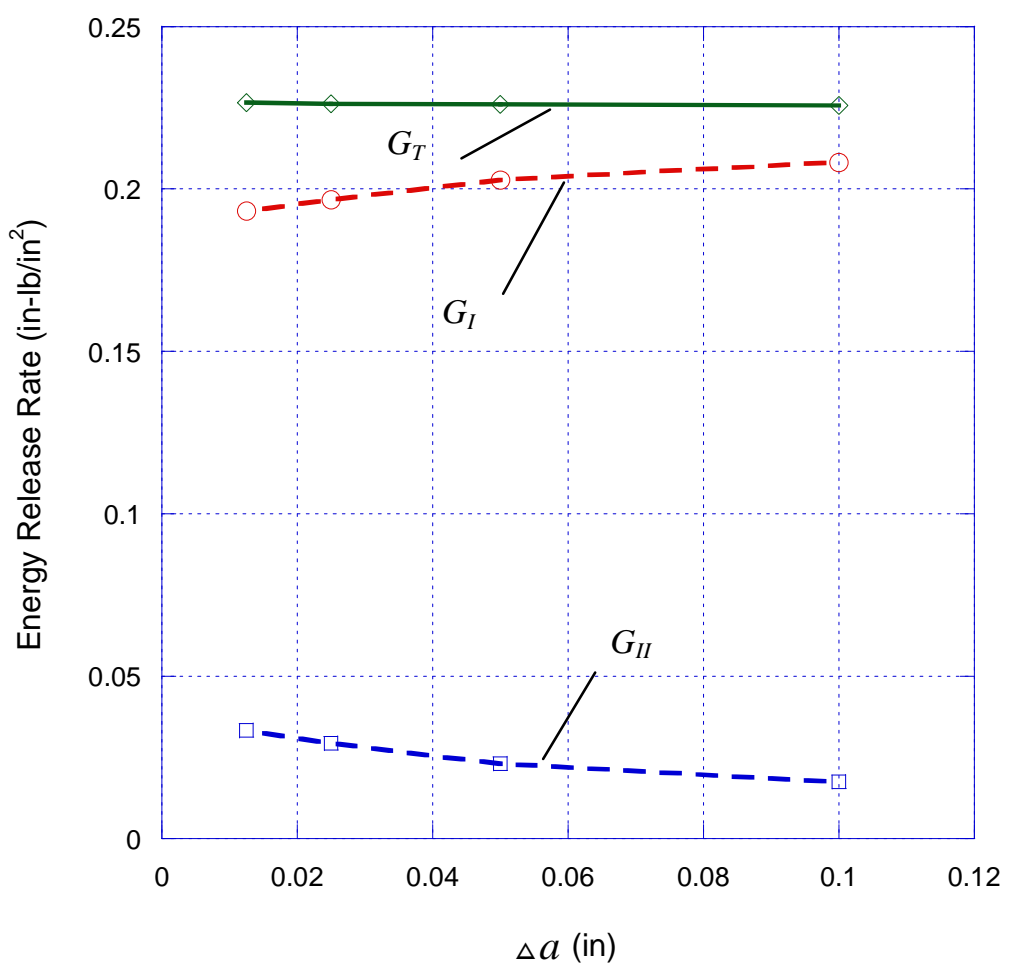

Fig. 4 Energy release rates for AL/(0-deg Glass/Epoxy) laminate.

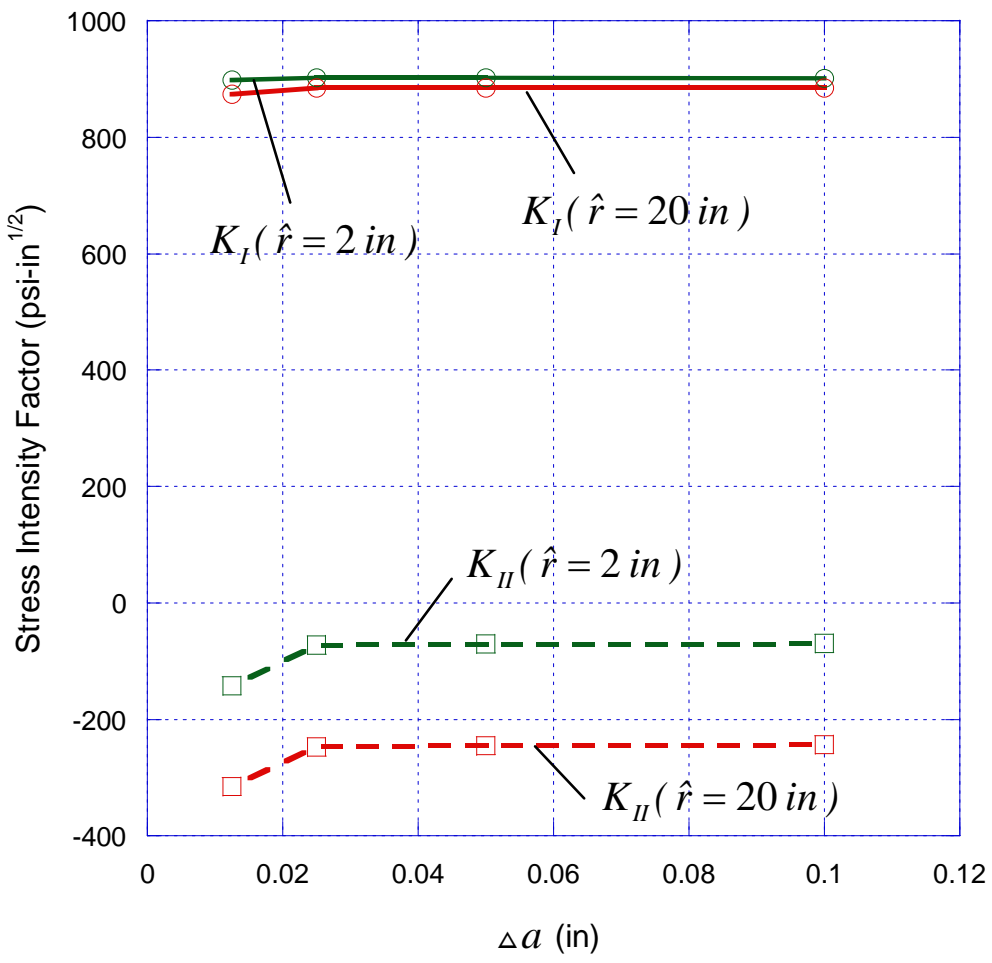

Fig. 5 Stress intensity factors for $\mathrm{AL} /(0-\mathrm{deg}$ Glass/Epoxy) laminate with relative crack-tip displacements at one element away from the crack tip. 


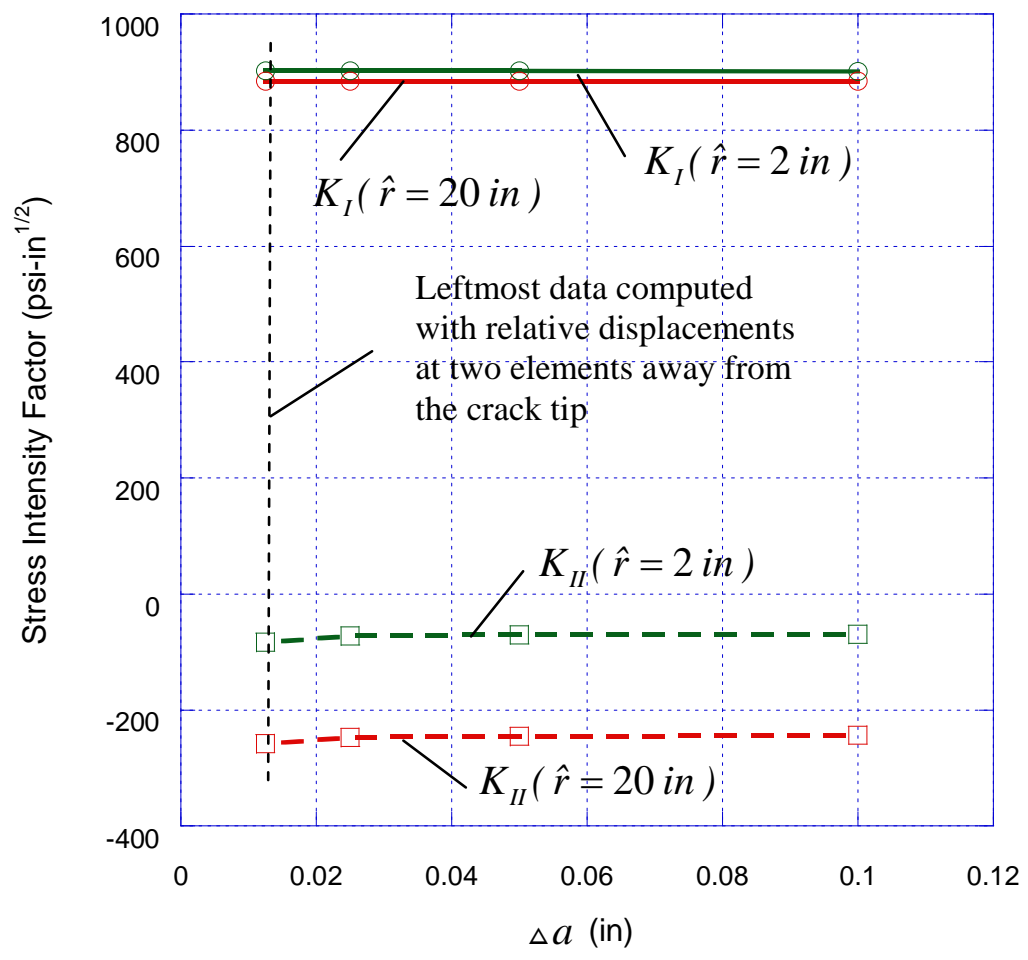

Fig. 6 Stress intensity factors for AL/(0-deg Glass/Epoxy) laminate (leftmost data computed with relative crack-tip displacements at two elements away from the crack tip).

stress intensity factors computed by using one reference length can easily be converted to another reference length with Eq. (2).

The energy release rates computed for the models with glass fibers in the 90-deg direction are plotted in Fig. 7. $G_{T}$ is well defined, however, $G_{I}$ and $G_{I I}$ are nonconvergent with mesh refinement and can cross each other. This indicates that using $G_{I}$ and $G_{I I}$ in an interfacial crack failure criterion may be problematic. The individual stress intensity factors for models with various $\triangle a$ were obtained by the displacement ratio method. Figure 8 shows that the individual stress intensity factors are independent of mesh sizes for $\Delta a \geq 0.025 \mathrm{in}$. The stress intensity factor $K_{I I}$ at $\Delta a=0.0125 \mathrm{in}$. is less than those of the larger mesh size models. As discussed for the 0-deg case, the oscillatory displacement fields very near the crack-tip may not be accurately predicted using the ABAQUS ${ }^{\odot}$ CPE8 elements. Again, by taking the relative crack surface displacements at a distance of 0.025 in. (two elements) away from the crack tip, the values of the stress intensity factors for the smallest mesh size model are nearly the same as those of the larger mesh size models as shown in Fig. 9. Note that the stress intensity factors for two reference length parameters are shown in Figs. 8 and 9. Again, Figs. 8 and 9 show that changing the reference length from $\hat{r}=2$ to $\hat{r}=20$ in. can result in the changes of the $K_{I}$ and $K_{I I}$ values and the mode mixity angle, $\psi_{12}=\tan ^{-1}\left(K_{I I} / K_{I}\right)$. 


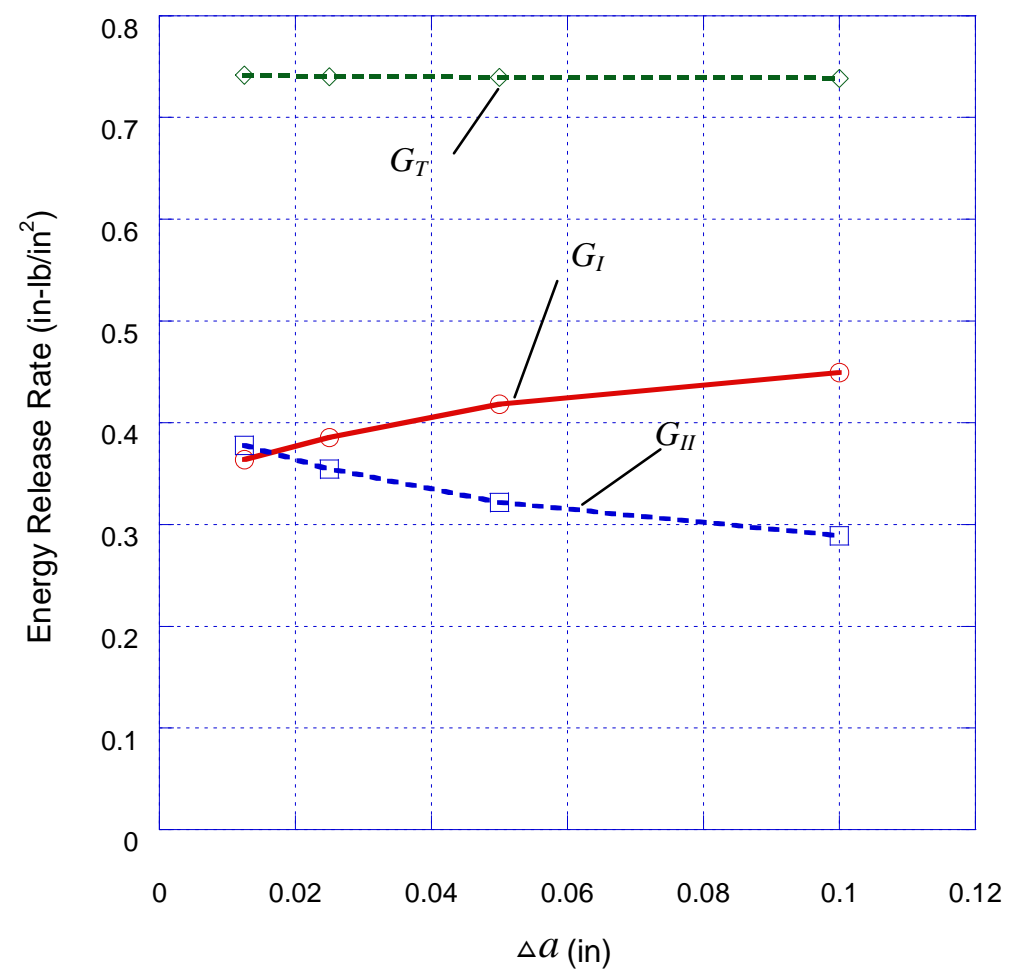

Fig. 7 Energy release rates for AL/(90-deg Glass/Epoxy) laminate.

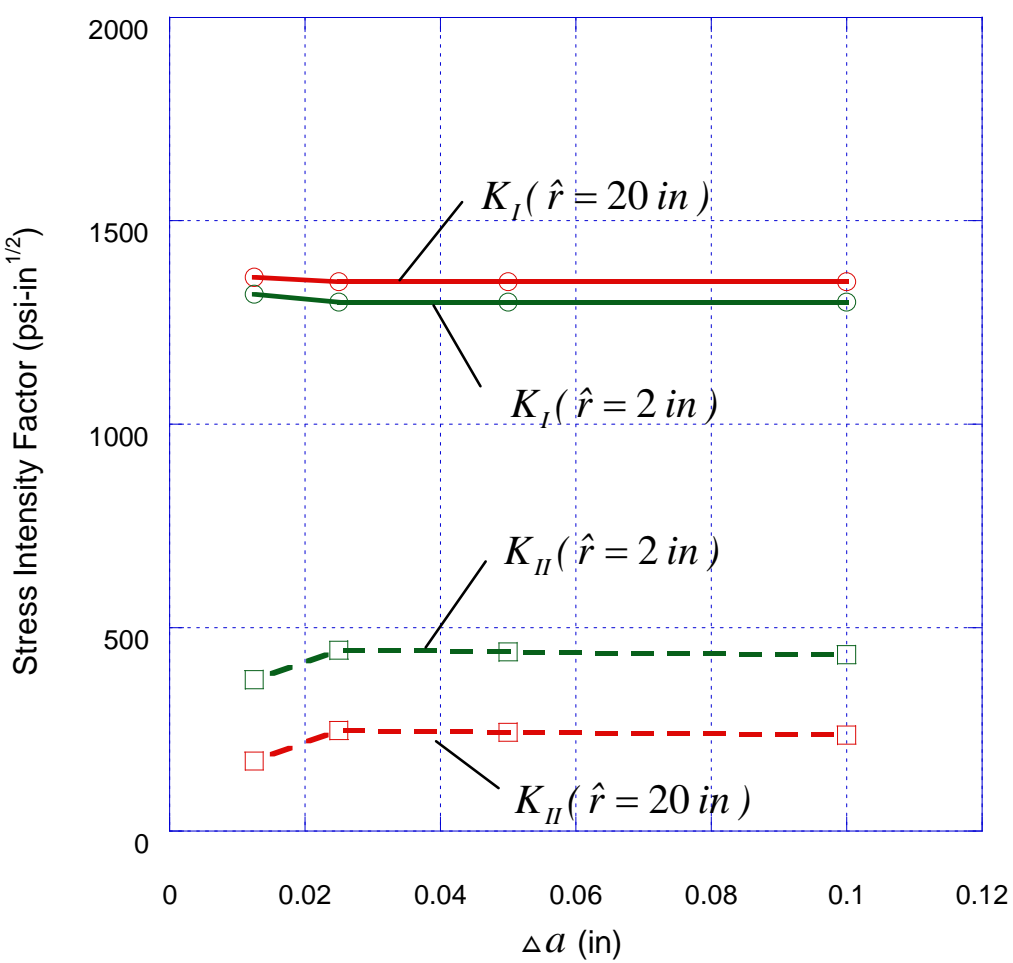

Fig. 8 Stress intensity factors for AL/(90-deg Glass/Epoxy) laminate with relative crack surface displacements at one element away from the crack tip.

10

American Institute of Aeronautics and Astronautics 


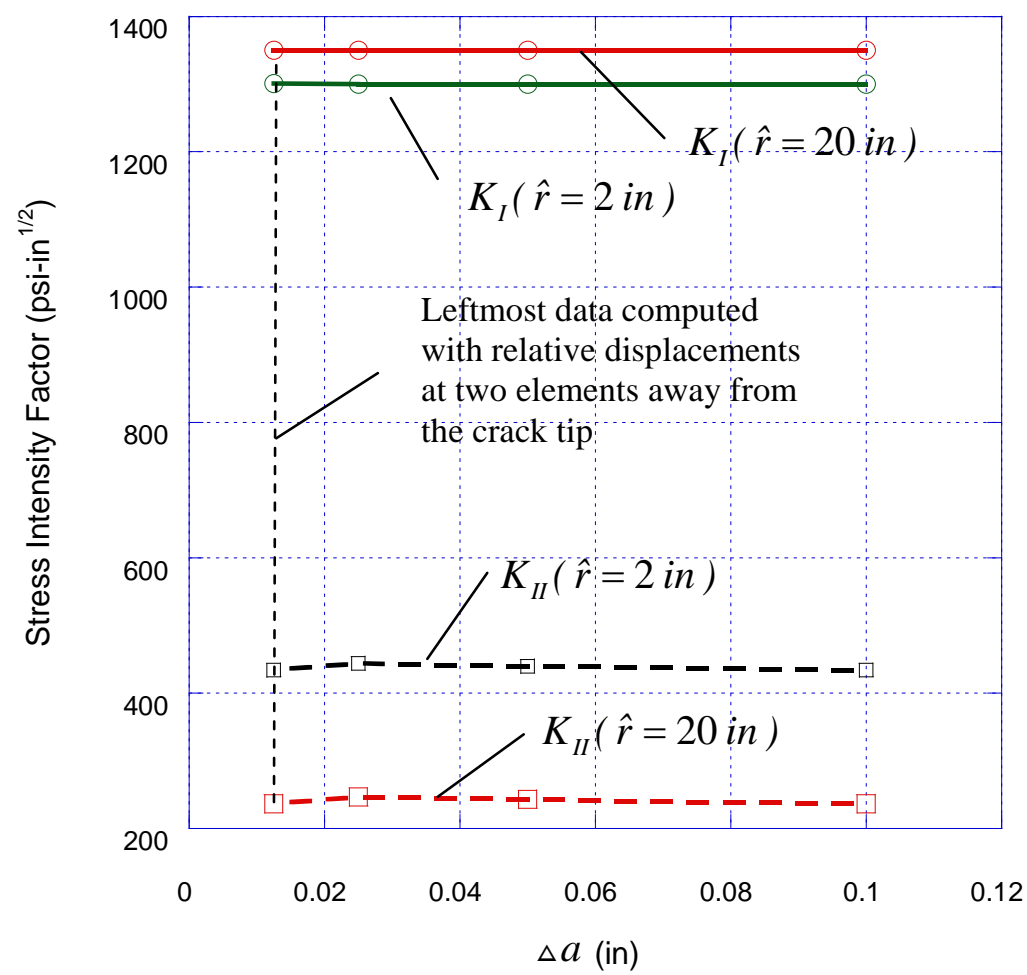

Fig. 9 Stress intensity factors for AL/(90-deg Glass/Epoxy) laminate (leftmost data computed with relative crack-tip displacements at two elements away from the crack tip).

\section{Generalized Plane Strain Finite Element Analyses}

A generalized plane strain finite element model for the FML shown in Fig. 2 was created and analyzed for studying the effects of varying the fiber angles of the Glass/Epoxy lay-ups on the energy release rates and the stress intensity factors. A modeling technique that can enforce the generalized plane strain deformations in the $x_{1}-x_{2}$ plane is used. The finite element model, created by extruding the mesh pattern in Fig. 3 along the $x_{3}$-direction, contains only one layer of brick elements, $0.05 \mathrm{in}$. in the $x_{3}$-direction. The 3D ABAQUS ${ }^{\odot}$ C3D8I finite element model has element size $\Delta a=0.025$ in. around the crack tip. To ensure no variation of strains in the $x_{3}$-direction, the corresponding nodes in the two parallel surfaces perpendicular to the $x_{3}$-direction are constrained to have the same $x_{3}$-displacements. The loading conditions and boundary conditions are the same as those shown in Fig. 3.

Seven finite element analyses were performed for the models with different lay-ups of the Glass/Epoxy part. These lay-ups are $\left[0_{4}\right],[+15 /-15]_{\mathrm{s}},[+30 /-30]_{\mathrm{s}},[+45 /-45]_{\mathrm{s}},[+60 /-60]_{\mathrm{s}},[+75 /-75]_{\mathrm{s}}$, and $\left[90_{4}\right]$. Because of the use of 3D solid elements, the engineering constants associated with the three principal material directions of each orthotropic ply and the ply orientation can be directly assigned to each element. The energy release rates versus layup angles for the interfacial crack are plotted in Fig. 10. The values of $G_{I}, G_{I I}$, and $G_{T}$ increase monotonically due to the increasing of flexibility of the Glass/Epoxy part. Note all $G_{I I I}$ values are near zero. The total energy release rates shown in Fig. 10 were used in the displacement ratio method to obtain the stress intensity factors. Figure 11 plots the $K_{I}, K_{I I}$, and $K_{I I I}$ versus lay-up angles, which represent the various lay-ups. For example, angle (+/-45 Deg) on the abscissa is the [+45/-45] S lay-up. $K_{I}$ increases as the fiber angle increases, and the curve becomes flat for fiber angles greater than 60-deg. The maximum values of $K_{I I}$ and $K_{I I I}$ occur at the 45-deg angle. Note that for the same lay-up, a zero $G_{I I I}$ value in Fig. 10 does not correspond to a zero $K_{I I I}$ value in Fig. 11, indicating that there is not a one to one relationship between $K_{I I I}$ and $G_{I I I}$. Each stress intensity factor is coupled with all three individual modes of strain energy release rates. $K_{I I}$ becomes positive for the fiber angles greater than 25-deg and nearly constant for the fiber angles greater than 45 -deg. The mode mixity angles can then be determined with the 
computed $K_{I}, K_{I I}$, and $K_{I I I}$ values and be used in Eq. (9) for predicting the mixed mode delamination failure at the interface between the aluminum part and the Glass/Epoxy part shown in Fig. 2.

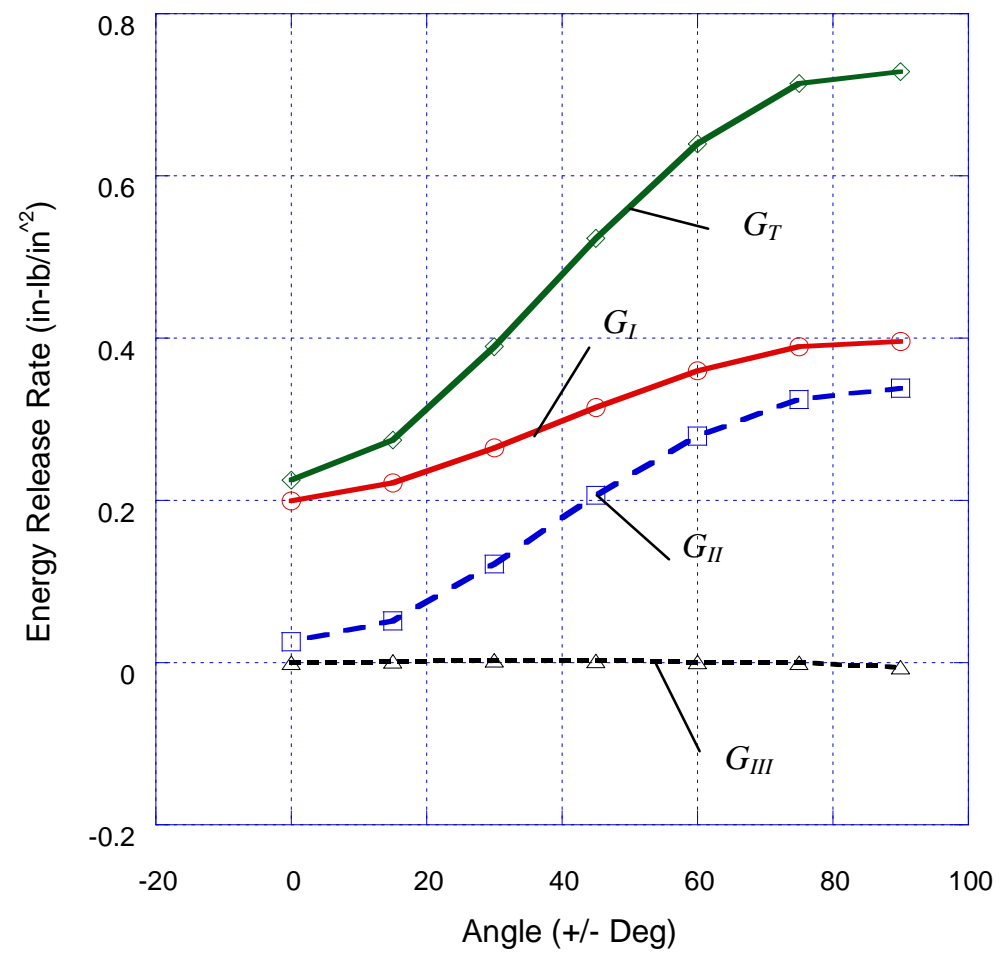

Fig. 10 Energy release rates for FMLs with four symmetric fiber layers.

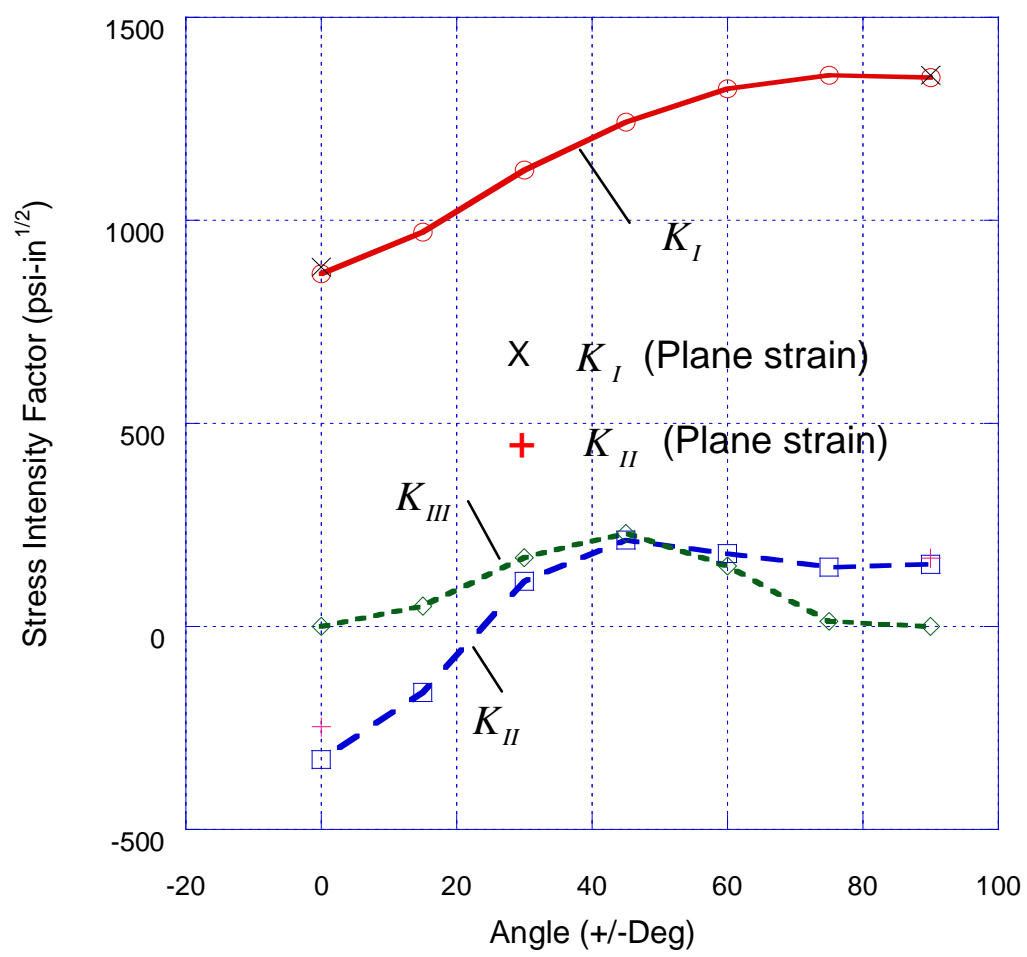

Fig. 11 Stress intensity factors for FMLs with four symmetric fiber layers. 


\section{B. FML with Multiple Interfacial Cracks under In-Plane Tension Loading}

A CentrAL-like configuration with fractured aluminum layers and multiple delaminations under in-plane tension loading shown in Fig. 12a was analyzed. This configuration is selected to demonstrate that the analysis technique developed here can also be applied to predict the failure of multiple interfacial delaminations in the CentrAL FMLs. Note that all the fiber layers are intact. Figure 12b shows a cross-section of $0.116 \mathrm{in}$. in length and $0.176 \mathrm{in}$. in height in the $x_{1}-x_{2}$ plane. The two outer aluminum layers are twice as thick as the internal aluminum layers. The thicknesses of both the aluminum and Glass/Epoxy layers are shown in the figure. Near the cracks in the aluminum layers, short delaminations exist between the aluminum and the Glass/Epoxy layers. This configuration was loaded with a uniform in-plane tension loading that can induce shear deformations at the interfacial crack tips. By exploiting symmetry, a finite element model of the upper right quadrant was created and analyzed. Figure 13 shows the symmetry boundary conditions, $\mathrm{u}=0$ at the left ends of the Glass/Epoxy layers and $\mathrm{v}=0$ at the bottom edge of the model. A uniform displacement, $\mathrm{u}=0.0015 \mathrm{in}$. was applied at the right edge. This upper right quadrant contains four delamination cracks and was modeled with ABAQUS 8-node plane strain, CPE8, elements. The strain energy release rates and the stress intensity factors were computed for each delamination crack.

The deformed configuration shown in Fig. 13 reveals that the crack opening displacements are gradually decreasing from the outermost crack, Crack \#1, to the innermost crack, Crack \#4. This indicates that Crck \#1 may have the highest Mode I energy release rate and stress intensity factor. The values of the energy release rates $G_{I}, G_{I I}$

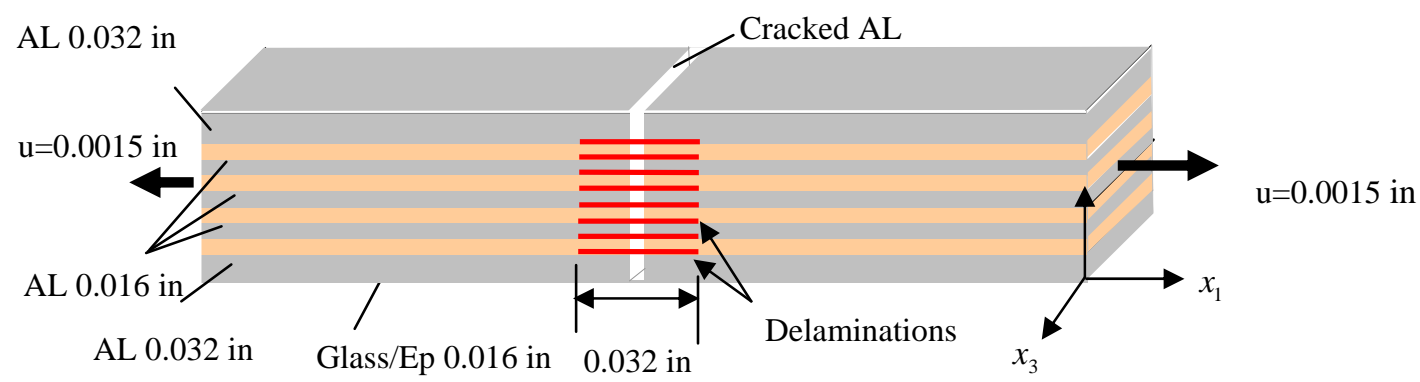

(a) 3D view of the damaged FML (not to scale)

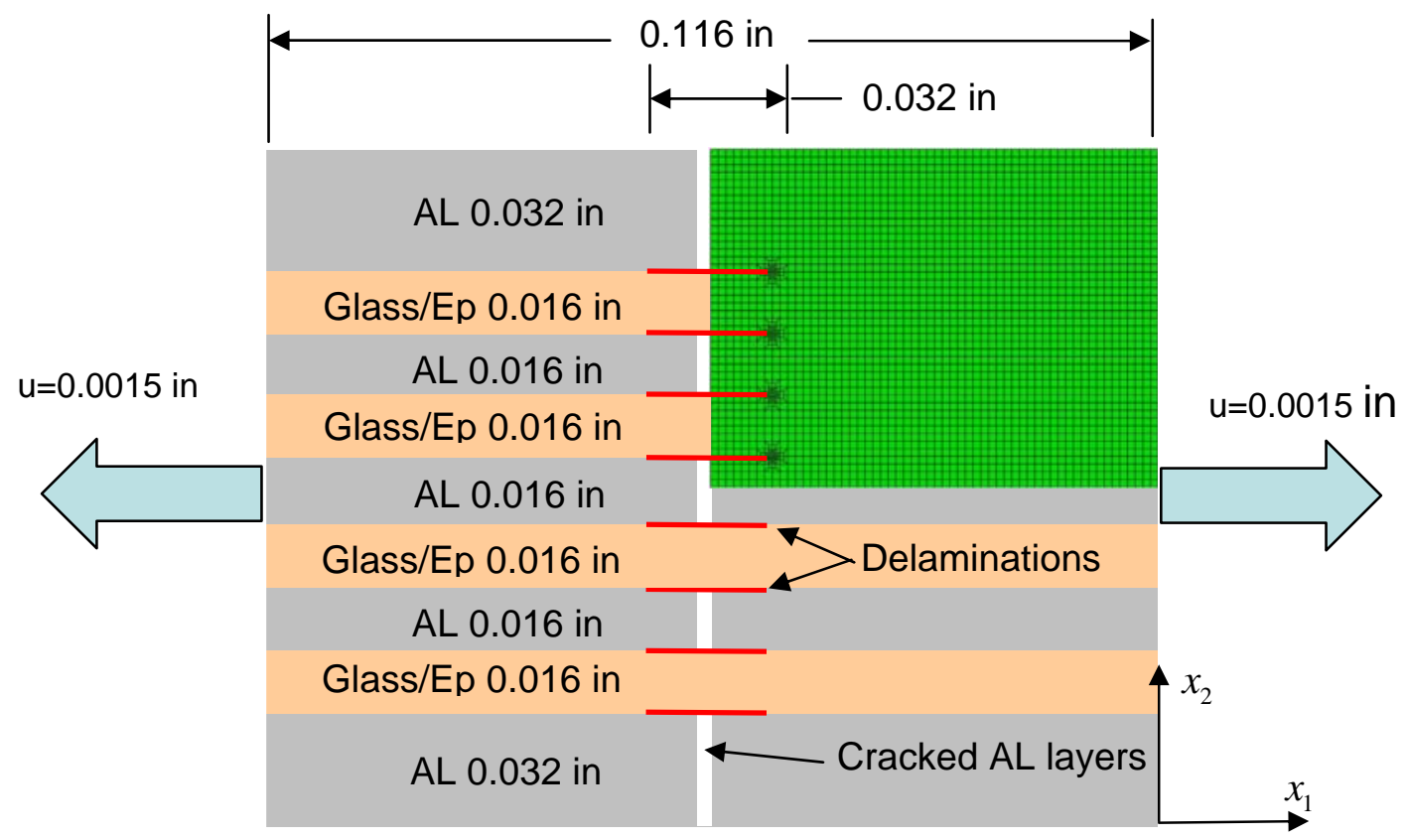

(b) Plane strain model ( $x_{1}-x_{2}$ plane)

Fig. 12 Fiber metal laminate with multiple cracked aluminum layers and delaminations. 
, and $G_{T}$ for each crack are plotted in Fig. 14. The mode separation method presented in Section II.C is used to compute the stress intensity factors for each delamination crack. Two different reference length parameters, $\hat{r}=$ 0.032 in. and 2.0 in., are used for computing the stress intensity factors to investigate whether the computed stress intensity factors and the mode mixity angles are dependent on the reference parameter used. The values of the stress intensity factors are plotted in Figs. 15a and 15b, and the values of the mode mixity angles are plotted in Fig. 15c. Figures 14 and 15 show that Mode-II is the dominant mode under the in-plane tensile load, $G_{I I} \gg G_{I}$ and $K_{I I} \gg K_{I}$. The Mode-I energy release rate data in Fig. 14 and the $K_{I}$ stress intensity factor data in Fig. 15a both show that the greatest values occur at Crack \#1.

The total energy release rates and mode mixity angles for the delamination cracks, obtained by using the two different reference length parameters, are shown in Fig. 16. These total energy release rates and mode mixity angles can be used with the mixed mode failure criterion, Eq. (9), to determine delamination failure. For the same crack, the mode mixity angle obtained by using one reference length parameter can be quite different from using another reference length parameter as shown in Figs. 15 and 16. As a result, it is necessary to provide the reference length parameter with the stress intensity factors reported. The user can convert the stress intensity factors to other reference length parameters by using Eq. (2).

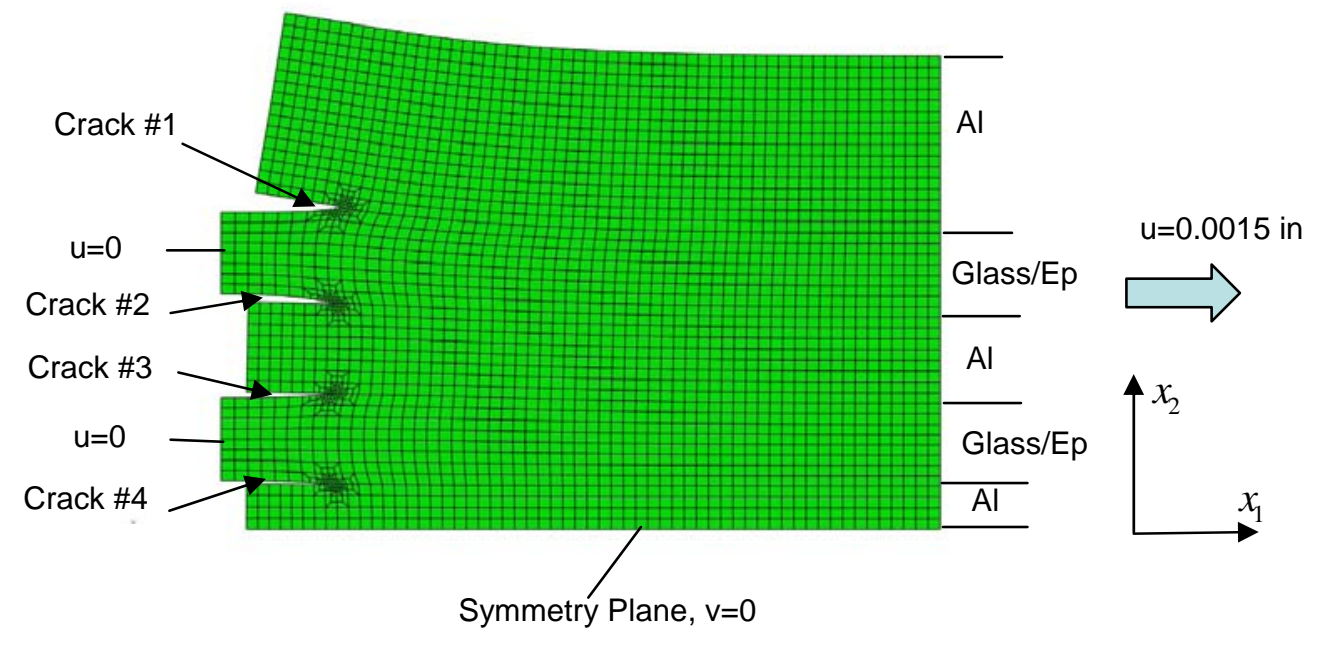

Fig. 13 Deformed shape and boundary conditions.

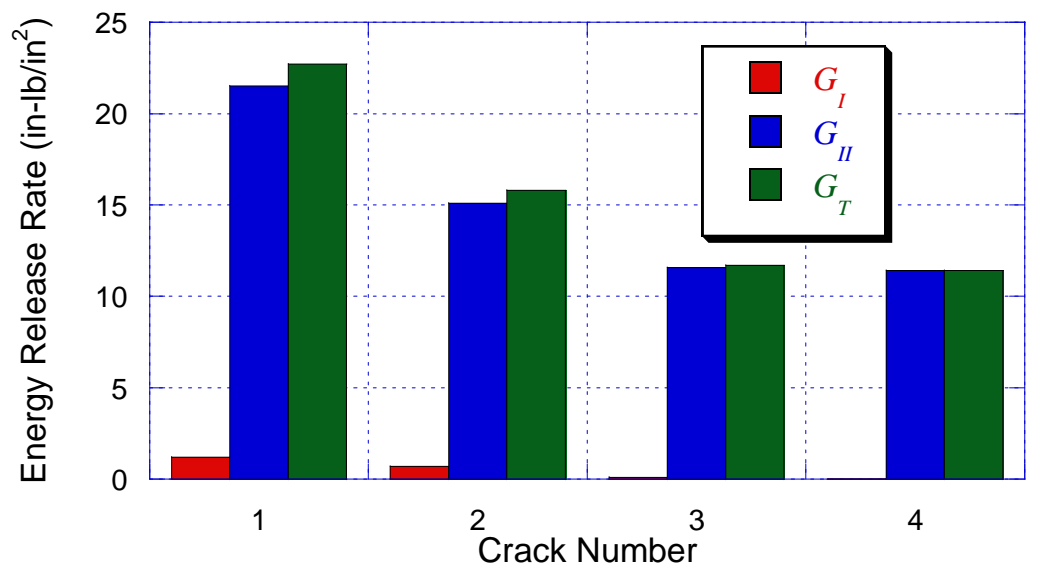

Fig. 14 Energy release rates for multiple cracks in the FML. 


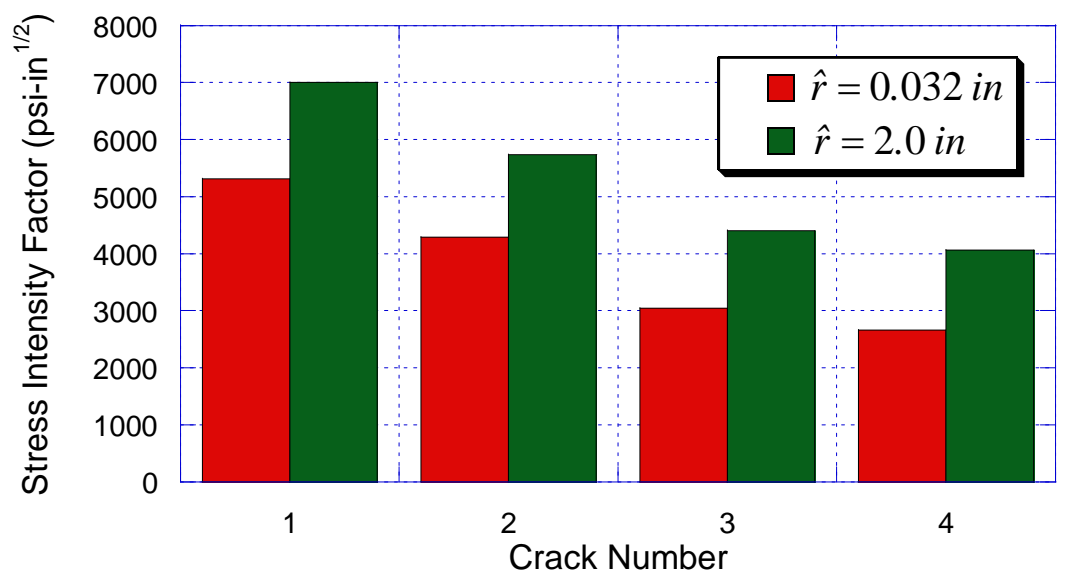

(a) $K_{I}$ stress intensity factor

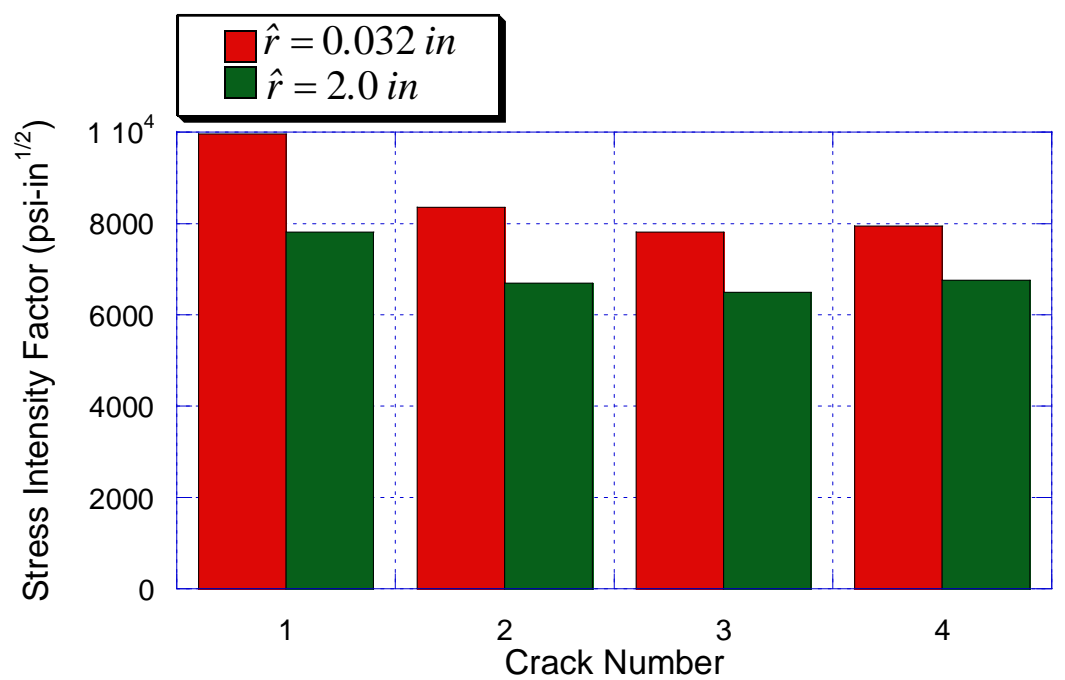

(b) $K_{I I}$ stress intensity factors

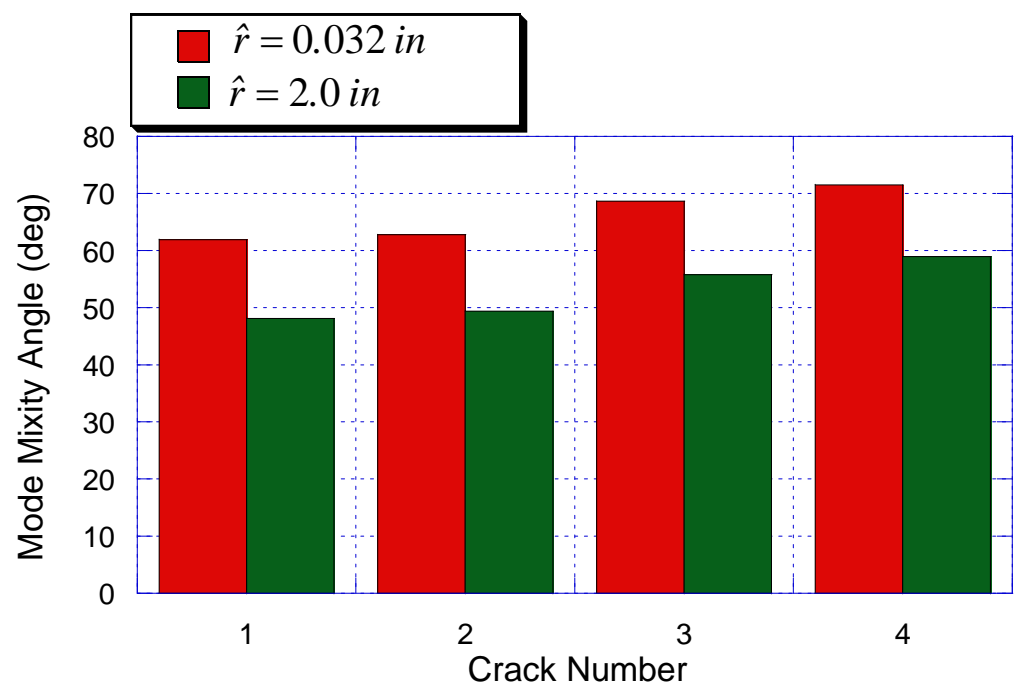

(c) Mode mixity angles

Fig. 15 Stress intensity factors and mode mixity angles for a multiple cracked FML. 


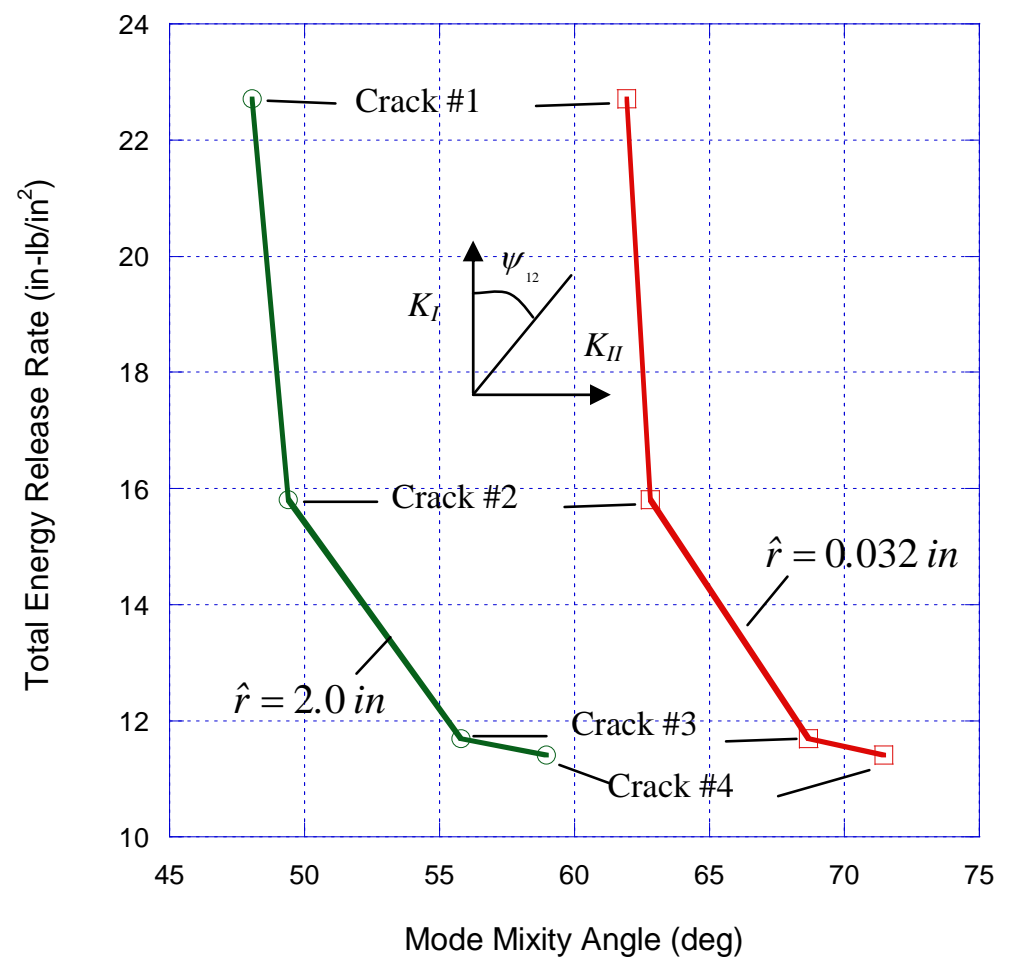

Fig. 16 Total energy release rates and mode mixity angles for multiple cracks in FML.

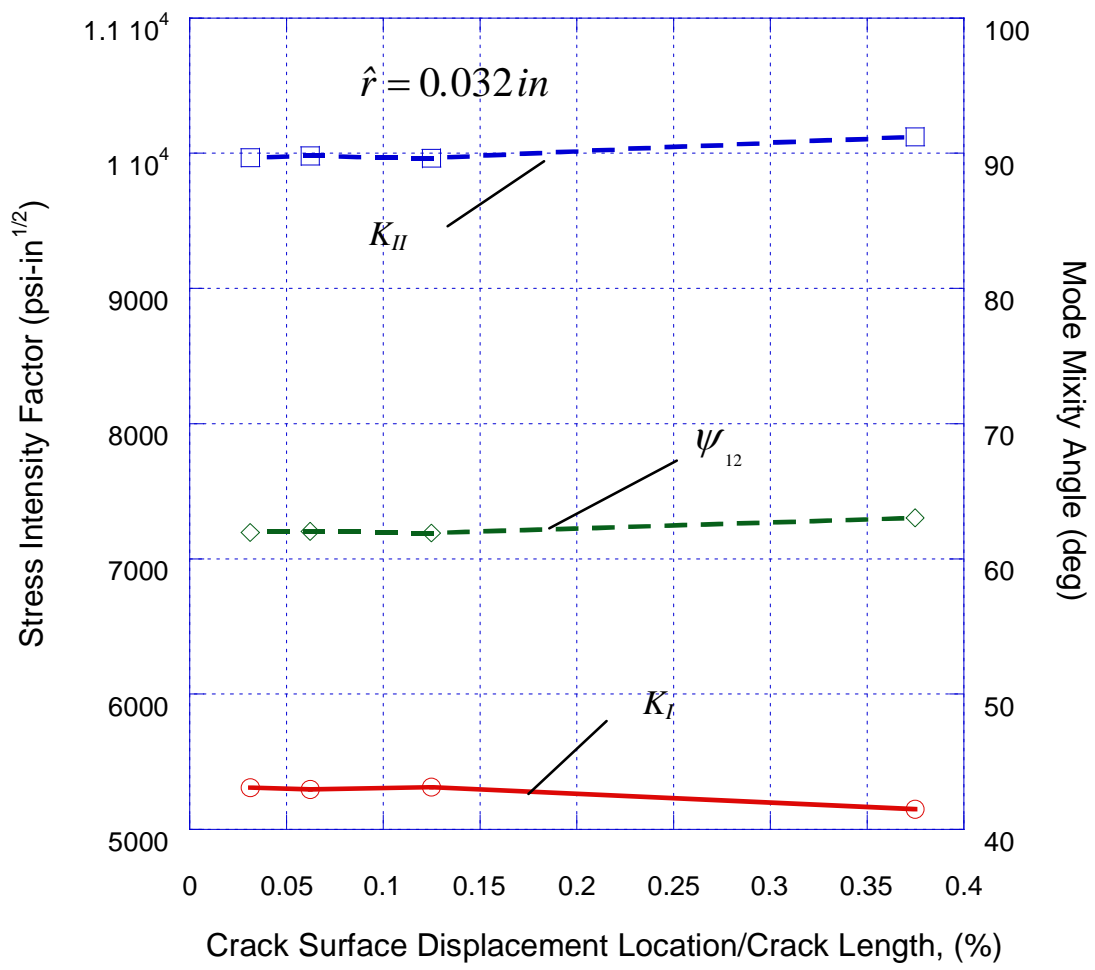

Fig. 17 Stress intensity factors predicted by using relative crack surface displacements at various distances from the crack tip. 
The discussion in Section III.A.1 notes that the stress intensity factors obtained by the displacement ratio method may be inaccurate if the relative crack surface displacements are taken at a location very near the crack tip. It can be useful to understand the sensitivity between the predicted stress intensity factors and the locations (distances from the crack tip) for taking the relative crack surface displacements. Relative crack surface displacements at four different locations taken from the finite element analysis results were used in the displacement methods to obtain the stress intensity factors. These locations are at 3.125\%, 6.25\%, $12.55 \%$ and $37.5 \%$ of the delamination crack length from the crack tip. Note that the delamination crack length in the finite element model is 0.016 in. (see Figs. 12). Stress intensity factors computed with these relative crack surface displacements for four different locations are plotted in Fig. 17. The stress intensity factors and mode mixity angles obtained by using the relative crack surface displacements at the first three locations are similar. Using the relative crack surface displacements at $37.5 \%$ of the delamination length, the stress intensity factors are all less than $3 \%$ from the previous values. This may indicate that the relative crack surface displacements can be taken at a distance of many elements away from the crack tip, and the computed stress intensity factors are insensitive to the location where the relative crack surface displacements are taken.

\section{Concluding Remarks}

A procedure for obtaining stress intensity factors based on the total strain energy release rate, material parameters from anisotropic elasticity, and the near-tip relative crack surface displacement ratios was implemented for calculating the stress intensity factors of interfacial cracks in FMLs. This study showed that mesh-sizeindependent stress intensity factors are obtainable if the relative crack surface displacements are not taken at a location very near the crack tip. By using these stress intensity factors, mesh-size-independent mode mixity angles can be obtained and used with the experimentally determined critical total energy release rate for defining a mixed mode fracture criterion. The stress intensity factors can be obtained very efficiently by the displacement method because its inputs, the total energy release rate and the relative crack surface displacements, can be provided by a single finite element analysis.

Various FML configurations with single or multiple delaminations and different loading conditions were investigated and presented in the paper. This study found that fiber orientations can significantly affect the Mode-I stress intensity factors for a FML configuration with an opening mode loading. For a CentrAL-like configuration with fractured aluminum layers and multiple delaminations, both the $K_{I}$ and $K_{I I}$ stress intensity factors have their highest values at the outermost crack and the lowest values at the innermost crack, gradually decreasing the values of stress intensity factors from the outermost crack toward the innermost crack.

This study also found that the selection of reference length can affect the magnitudes and the mode mixity angles of the stress intensity factors; thus, it is important to report the reference length used for calculating the reported stress intensity factors. The last finding is that the relative crack surface displacements can be taken at a distance of many elements away from the crack tip. The stress intensity factors computed by the displacement ratio method are insensitive to the locations where the relative crack surface displacements were taken.

\section{Appendix - Stroh Formalism, Bimaterial Eigenequation, and Bimaterial Parameters}

This appendix provides some background information related to how the eigenvalues and eigenvectors used in Eq. (1) are obtained. The eigenequation was derived by $\mathrm{Hwu}^{21}$ based on the sextic formalism of Stroh. ${ }^{9}$ A brief introduction of Stroh formalism is included here. For a bimaterial interfacial crack, Stroh formalism was used to establish the impedance matrices ${ }^{21} \mathbf{M}$ for the upper part material, Material \#1, and the lower part material, Material \#2, that define the crack plane. These impedance matrices were then used to form the bimaterial matrix $\mathbf{M}^{*}$ that was used to establish the bimaterial eigenequation. ${ }^{21}$ The forms of the matrices $\mathbf{M}$ and $\mathbf{M}^{*}$ will be given later.

The stress-strain relations of an anisotropic system are given by

$$
\sigma_{i j}=C_{i j k s} u_{k, s}
$$

where $\sigma_{i j}$ are the stresses, $u_{k, s}$ are the strains, and $C_{i j k s}$ are the stiffnesses. The equations of equilibrium are given as

$$
C_{i j k s} u_{k, s j}=0
$$


For a generalized plane strain deformation in which $u_{k}(k=1,2,3)$ only depend on $x_{1}$ and $x_{2}$ of a fixed rectangular coordinate system, the displacements can be assumed as ${ }^{9,22,23}$

$$
u_{k}=a_{k} f(z)
$$

where $z=x_{1}+p x_{2}$. p and $a_{k}$ are unknown constants to be determined. The equations of equilibrium can be rewritten as

$$
C_{i j k s}\left(\delta_{j 1}+p \delta_{j 2}\right)\left(\delta_{s 1}+p \delta_{s 2}\right) a_{k}=0
$$

or

$$
\left\{C_{i 1 k 1}+p\left(C_{i 1 k 2}+C_{i 2 k 1}\right)+p^{2} C_{i 2 k 2}\right\} a_{k}=0
$$

where $\delta_{i j}=\left\{\begin{array}{l}1.0, i=j \\ 0.0, i \neq j\end{array}\right.$ denotes the Kroneker delta. This can be written in matrix notation as

$$
\left\{\mathbf{Q}+p\left(\mathbf{R}+\mathbf{R}^{T}\right)+p^{2} \mathbf{T}\right\} \boldsymbol{a}=0
$$

where $\mathbf{Q}, \mathbf{R}$, and $\mathbf{T}$ are $3 \times 3$ matrices whose components are

$$
Q_{i k}=C_{i 1 k 1}, R_{i k}=C_{i 1 k 2}, T_{i k}=C_{i 2 k 2} \quad(i, k=1,2,3)
$$

Eq. (A6) can be rewritten as

$$
(\mathbf{Q}+p \mathbf{R}) \boldsymbol{a}=-p\left(\mathbf{R}^{T}+p \mathbf{T}\right) \boldsymbol{a}
$$

By introducing a new vector $\mathbf{b}=\left(\mathbf{R}^{T}+p \mathbf{T}\right) \boldsymbol{a}$ and using Eq. (A8), the following expression can be derived,

$$
\mathbf{b}=\left(\mathbf{R}^{T}+p \mathbf{T}\right) \boldsymbol{a}=-\frac{1}{p}(\mathbf{Q}+p \mathbf{R}) \boldsymbol{a}
$$

Equation (A8) can be recast as a standard eigenvalue problem,

$$
\mathbf{N} \xi=p \xi
$$

in which

$$
\mathbf{N}=\left[\begin{array}{cc}
-\mathbf{T}^{-1} \mathbf{R}^{T} & \mathbf{T}^{-1} \\
\mathbf{R T}^{-1} \mathbf{R}^{T}-\mathbf{Q} & -\mathbf{R T}^{-T}
\end{array}\right] \text {, and } \boldsymbol{\xi}=\left(\begin{array}{l}
\mathbf{a} \\
\mathbf{b}
\end{array}\right)
$$

Six eigenvalues of $p$ and six associated eigenvectors of $\xi$ can be obtained. It can be shown that $p$ cannot be real if the strain energy is positive. ${ }^{23}$ Therefore, three pairs of complex conjugate $p$ and three pairs of complex conjugate $\xi$ are as follows

$$
p_{i+3}=\bar{p}_{i} \text { and } \xi_{i+3}=\bar{\xi}_{i}(i=1,2,3)
$$


Note that $p_{i}(i=1,3)$ have positive imaginary parts and their associated eigenvectors, $\xi_{i}=\left(\begin{array}{l}a_{i} \\ b_{i}\end{array}\right)$, are used to define two matrices, $\mathbf{A}$ and $\mathbf{B}$.

$$
\mathbf{A}=\left[\begin{array}{lll}
\mathbf{a}_{1} & \mathbf{a}_{2} & \mathbf{a}_{3}
\end{array}\right], \mathbf{B}=\left[\begin{array}{lll}
\mathbf{b}_{1} & \mathbf{b}_{2} & \mathbf{b}_{3}
\end{array}\right]
$$

The bimaterial matrix $\mathbf{M}^{*}$ (Ref. 21) is defined with the impedance matrices, $\mathbf{M}_{\mathbf{i}}(\mathrm{i}=1,2)$ as

$$
\mathbf{M}^{*}=\mathbf{M}_{1}^{-1}+\overline{\mathbf{M}}_{2}^{-1}=\mathbf{i}\left(\mathbf{A}_{1} \mathbf{B}_{1}^{-1}-\overline{\mathbf{A}}_{2} \overline{\mathbf{B}}_{2}^{-1}\right)=-\mathbf{i}(\mathbf{W}+\mathbf{i D})
$$

where $\mathbf{W}$ and $\mathbf{D}$ are real matices and are related to Barnet-Lothe's tensors $\mathbf{S}$ and $\mathbf{L}$ (Ref. 24) as

$$
\mathbf{W}=\mathbf{S}_{1} \mathbf{L}_{1}-\mathbf{S}_{2} \mathbf{L}_{2}, \quad \mathbf{D}=\mathbf{L}_{1}^{-1}+\mathbf{L}_{2}^{-1}
$$

In this study, the upper part, MAT\#1, is isotropic, the $\mathbf{A}_{1} \mathbf{B}_{1}^{-1}$ term may be computed directly from the explicit expressions of $\mathbf{S}_{\mathbf{1}}$ and $\mathbf{L}_{\mathbf{1}}$ (Ref. 22) to avoid the problems related to the degenerate material by writing the $\mathbf{A}_{1} \mathbf{B}_{1}^{-1}$ $\mathrm{as}^{22}$

$$
\mathbf{A}_{1} \mathbf{B}_{1}^{-1}=-\left(\mathbf{S}_{1} \mathbf{L}_{1}^{-1}+\mathbf{i} \mathbf{L}_{1}^{-1}\right)
$$

where

$$
\mathbf{S}_{1}=\frac{1-2 v}{2(1-v)}\left[\begin{array}{ccc}
0 & -1 & 0 \\
1 & 0 & 0 \\
0 & 0 & 0
\end{array}\right], \quad \mathbf{L}_{1}=\mu\left[\begin{array}{ccc}
\frac{1}{1-v} & 0 & 0 \\
0 & \frac{1}{1-v} & 0 \\
0 & 0 & 1
\end{array}\right]
$$

$v$ is the poisson's ratio, and $\mu$ is the shear modulus.

For FMLs, the $\mathbf{D}$ and $\mathbf{W}$ can be expressed as the following forms ${ }^{8}$

$$
\mathbf{D}=\left[\begin{array}{ccc}
D_{11} & 0 & D_{13} \\
0 & D_{22} & 0 \\
D_{13} & 0 & D_{33}
\end{array}\right], \quad \mathbf{W}=\left[\begin{array}{ccc}
0 & -W_{21} & 0 \\
W_{21} & 0 & -W_{32} \\
0 & W_{32} & 0
\end{array}\right]
$$

The $\boldsymbol{\Lambda}$ in Eq. (1) is obtained from the following bimaterial eigenequation formulated by Hwu. ${ }^{21}$

$$
\left(\mathbf{M}^{*}+\mathrm{e}^{2 i \pi \delta} \overline{\mathbf{M}}^{*}\right) \boldsymbol{\Lambda}=\mathbf{0}
$$

The explicit solution of $\delta$ was given by Ting ${ }^{25}$ as

$$
\delta_{\alpha}=-\frac{1}{2}+i \varepsilon_{\alpha}, \alpha=1,2,3
$$

where

$$
\varepsilon_{1}=\varepsilon=\frac{1}{2 \pi} \ln \frac{1+\beta}{1-\beta}, \varepsilon_{2}=-\varepsilon, \varepsilon_{3}=0,
$$


and

$$
\beta=\left[-\frac{1}{2} \operatorname{tr}\left(\mathbf{W D}^{-1}\right)^{2}\right]^{1 / 2}
$$

where tr stands for the trace of a matrix and $\varepsilon$ is called the oscillation index. The three eigenvectors of Eq. (A18) are $\boldsymbol{\Lambda}=\left[\begin{array}{lll}\lambda_{1} & \lambda_{2} & \lambda_{3}\end{array}\right]$ and are normalized by $\overline{\boldsymbol{\Lambda}}^{\mathrm{T}} \mathbf{D} \boldsymbol{\Lambda}=\mathbf{I}$. Using the $\mathbf{W}$ and $\mathbf{D}$ forms shown in Eq. (A17), the $\beta$ and $\Lambda$ can be explicitly expressed as ${ }^{8}$

$$
\begin{gathered}
\beta=\sqrt{\frac{W_{21} a_{f}+W_{32} d_{f}}{D_{22} e_{f}}} \\
\boldsymbol{\Lambda}=\left[\begin{array}{ccc}
-i a_{f} / b_{f} c_{f} & i a_{f} / b_{f} c_{f} & W_{32} / \sqrt{b_{f} \beta D_{22}} \\
1 / c_{f} & 1 / c_{f} & 0 \\
i d_{f} / b_{f} c_{f} & -i d_{f} / b_{f} c_{f} & W_{21} / \sqrt{b_{f} \beta D_{22}}
\end{array}\right]
\end{gathered}
$$

where the coefficients $a_{f}, b_{f}, c_{f}, d_{f}$, and $e_{f}$ are given as

$$
\begin{aligned}
& a_{f}=W_{21} D_{33}+W_{32} D_{13} \\
& b_{f}=\beta\left(D_{11} D_{33}-D_{13}^{2}\right) \\
& c_{f}=\sqrt{2 D_{22}} \\
& d_{f}=W_{32} D_{11}+W_{21} D_{13} \\
& e_{f}=D_{11} D_{33}-D_{13}^{2}
\end{aligned}
$$

The coefficients $a_{f}, b_{f}, c_{f}, d_{f}$, and $e_{f}$, oscillation index $\varepsilon$, and components of $\mathbf{W}$ and $\mathbf{D}$ matrices are designated as bimaterial parameters which are used in Eqs. (1) to (8).

\section{Acknowledgments}

The author would like to acknowledge and appreciate the helpful technical discussions with Professor C. T. Sun of Purdue University, Dr. Edward C. Jih of Ford Motor Company, and Dr. Zhengwen Yang of Hill-Rom, Inc. and the valuable editorial comments provided by his colleagues Drs. Edward H. Glaessgen, James G. Ratcliffe, and Jonathan B. Ransom, and Mr. Frank J. Tsai.

\section{References}

${ }^{1}$ Roebroeks, G. H. J. J., Hooijmeijer, P. A., Kroon, E. J., and Heinimann, M. B., “The Development of CentrAL,” First International Conference on Damage Tolerance of Aircraft Structures, R. Benedictus, J. Schijve, R. C. Alderliesten, J. J. Homan (Eds.), September 25-28, 2007, Delft University of Technology, The Netherlands.

${ }^{2}$ Alderliesten, R. C., Fatigue Crack Propagation and Delamination Growth in Glare, PhD Thesis, Delft University of Technology, ISBN 90-407-2588-8, 2005.

${ }^{3}$ Suiker, A. J. and Fleck, N. A., “Crack Tunneling and Plane-Strain Delamination in Layered Solids,” International Journal of Fracture, Vol. 125, 2004, pp. 1-32.

${ }^{4}$ Sun, C. T. and Jih, C. J., “On Strain Energy Release Rate for Interfacial Cracks in Bimaterial Media,” Engineering Fracture Mechanics, Vol. 28, 1987, pp.13-20.

${ }^{5}$ Raju, I. S., Crews J. H., and Aminpour, M. A., "Convergence of Strain Energy Release Rate Components for EdgeDelaminated Composite Laminates,” Engineering Fracture Mechanics, Vol. 30, 1988, pp. 383-396.

${ }^{6}$ Chow, W. T. and Atluri, S. N., "Finite Element Calculation of Stress Intensity Factors for Interfacial Crack Using Virtual Crack Closure Integral,” Computational Mechanics, Vol. 16, 1995, pp. 417-425.

${ }^{7}$ Sun, C. T. and Qian, W., "The Use of Finite Extension Strain Energy Release Rates in Fracture of Interfacial Cracks,” International Journal of Solids and Structures, Vol. 34, 1997, pp. 2595-2609.

20

American Institute of Aeronautics and Astronautics 
${ }^{8}$ Qian, W. and Sun, C. T., "Calculation of Stress Intensity Factors for Interlaminar Cracks in Composite Laminates," Composites Science and Technology, Vol. 57, 1997, pp. 637-650.

${ }^{9}$ Stroh, A. N., "Dislocations and Cracks in Anisotropic Elasticity,” Philosophical Magazine, Vol. 7, 1958, pp. 625-646.

${ }^{10}$ Rybicki, E. F. and Kanninen, M. F., "A Finite Element Calculation of Stress Intensity Factors by a Modified Crack Closure Integral,” Engineering Fracture Mechanics, Vol. 9, 1977, pp. 931-938.

${ }^{11}$ Hwu, C., "Fracture Parameters for the Orthotropic Bimaterial Interface Cracks,” Engineering Fracture Mechanics, Vol. 45, 1993, pp. 89-97.

${ }^{12} \mathrm{Wu}$, K. C., "Stress Intensity Factors and Energy Release Rate for Interfacial Cracks between Dissimilar Anisotropic Materials,” ASME Journal of Applied Mechanics, Vol. 57, 1990, pp. 882-886.

${ }^{13}$ Rice, J. R., "Elastic Fracture Mechanics Concepts for Interfacial Cracks,” ASME Journal of Applied Mechanics, Vol. 55, 1988, pp. 98-103.

${ }^{14}$ Irwin, G. R., “Analysis of Stresses and Strains near the End of a Crack Traversing a Plate,” ASME Journal of Applied Mechanics, Vol. 24, 1957, pp. 361-364.

${ }^{15}$ Liechti, K. M. and Knauss, W. G., “Crack Propagation at Material Interfaces: II Experiments on Mode Interaction," Experimental Mechanics, Vol. 22, 1982, pp. 383-391.

${ }^{16}$ Suo, Z. and Hutchinson, J. W., "Sandwich Test Specimens for Measuring Interface Crack Toughness,” Materials Science and Engineering, A107, 1989, pp. 135-143.

${ }^{17}$ Charalambides, P. G., Cao, H. C., Lund, J., and Evans, A. G., "Development of a Test Method for Measuring the Mixed Mode Fracture Resistance of Bimaterial Interfaces,” Mechanics of Materials, Vol. 8, 1990, pp. 269-283.

${ }^{18}$ Bower, A. F., Applied Mechanics of Solids, http://solidmechanics.org/text/.

${ }^{19}$ Chang, P. Y. and Yang, J. M., “Modeling of fatigue crack growth in notched fiber metal laminates,” International Journal of Fatigue, Vol. 30, 2008, pp. 2165-2174.

${ }^{20}$ ABAQUS Analysis User’s Manual, Version 6.6, ABAQUS Inc., 2006.

${ }^{21}$ Hwu, C., "Explicit Solutions for Co-linear Crack Problems,” International Journal of Solids and Structures, Vol. 3, 1993, pp. 301-312.

${ }^{22}$ Ting, T. C. T., Anisotropic Elasticity, Oxford University Press, Inc., New York, New York, ISBN 0-19-507447-5, 1996.

${ }^{23}$ Eshelby, J. D., Read, W. T., and Shockley, W., “Anisotropic Elasticity with Applications to Dislocation Theory,” Acta Metallurgica, Vol. 1, 1953, pp. 251-259.

${ }^{24}$ Barnett, D. M. and Lothe, J., "Synthesis of the Sextic and the Integral Formalism for Dislocation, Green's Function and Surface Waves in Anisotropic Elastic Solids,” Physica Norvegica, Vol. 7, 1973, pp. 13-19.

${ }^{25}$ Ting, T. C. T., "Explicit Solution and Invariance of the Singularities at an Interface Crack in Anisotropic Composites," International Journal of Solids and Structures, Vol. 22, 1986, pp. 965-983. 